Cahiers Charlevoix

Études franco-ontariennes
Cahiers Charlevoix Études franco-ontariennes

or Crevenerix of

\title{
La chanson française de tradition orale en Ontario : données, tendances, état du répertoire
}

\section{Marcel Bénéteau}

Volume 12, 2018

URI : https://id.erudit.org/iderudit/1048919ar

DOI : https://doi.org/10.7202/1048919ar

Aller au sommaire du numéro

\section{Éditeur(s)}

Société Charlevoix

Presses de l’Université d'Ottawa

\section{ISSN}

1203-4371 (imprimé)

2371-6878 (numérique)

Découvrir la revue

Citer cet article

Bénéteau, M. (2018). La chanson française de tradition orale en Ontario : données, tendances, état du répertoire. Cahiers Charlevoix, 12, 149-215.

https://doi.org/10.7202/1048919ar
Résumé de l'article

L'étude de Marcel Bénéteau fait le bilan de la chanson traditionnelle française en Ontario et trace son évolution dans le temps et l'espace. L'auteur décrit le travail qu'il a entrepris pour déterminer en premier lieu en quoi consiste le répertoire de l'Ontario français ; à cette fin, il précise quelles sont ses balises historiques et géographiques (où et quand le répertoire s'est-il implanté sur le territoire ?) et ce qu'il comprend au juste (combien de chansons ? quelles chansons? quelles catégories de chansons ?). Pour ce faire, il a dû s'astreindre à dépouiller les divers fonds d'archives disponibles afin de repérer, identifier et cataloguer plus de 12000 versions de chansons recueillies en Ontario depuis la fin du XIX ${ }^{e}$ siècle, un travail de longue haleine qui a occupé l'auteur au cours des dix dernières années. Le résultat - premiers jalons d'un catalogue de la chanson traditionnelle française en Ontario - permet de décrire avec précision la richesse et la diversité du répertoire tout en tenant compte de ses particularités régionales et de ses apports au fonds commun que partagent les francophones des deux côtés de l'Atlantique. 


\section{La chanson française de tradition orale en Ontario : données, tendances, état du répertoire}

Marcel BéNÉTEAu

Département de folklore et ethnologie Université de Sudbury 


\section{SOMMAIRE}

1. ÉtAT DE LA QUESTION 151

Quelques définitions 155

Identification des chansons $\quad 157$

Les catégories Laforte $\quad 159$

I. Chansons en laisse 159

II. Chansons strophiques 161

III. Chansons en forme de dialogue 164

IV. Chansons énumératives 165

V. Chansons brèves 166

VI. Chansons sur les timbres 167

2. LE RÉPERTOIRE FRANCO-ONTARIEN 169

Analyse des répertoires régionaux 171

1. Le Nord 172

Les collections 173

2. L'Est ontarien 189

Les collections 189

3. Le sud de l'Ontario 194

Les collections 197

3. CONSIDÉRATIONS FINALES 207

$\begin{array}{ll}\text { CONCLUSION } & 214\end{array}$ 


\section{La chanson française de tradition orale en Ontario : données, tendances, état du répertoire}

\section{1. ÉTAT DE LA QUESTION}

L'identification et la classification des matériaux est nécessaire pour toute analyse raisonnée d'une série de données ; même s'il ne s'agit que d'une première étape, l'ordonnance systématique des éléments d'un corpus permet d'en dégager ses traits saillants, de tracer son évolution et de le comparer à d'autres corpus organisés selon des principes compatibles. La chanson de tradition orale chez les francophones de l'Ontario, bien qu'elle ait été le sujet de plusieurs études est quand même perçue de façon curieusement vague, autant par le grand public que par la communauté scientifique. On sait très bien qu'elle existe, qu'elle est vivante au sein des familles et des communautés, qu'elle a attiré l'attention de folkloristes comme Germain Lemieux. Mais le travail dans ce domaine a rarement été au-delà de la cueillette et de l'inventaire; la plupart des opinions émises à son sujet consistent en un mélange d'hypothèses, d'extrapolations et de généralisations faites à partir de corpus mieux définis, avec des tentatives de lier sa présence sur le terrain ontarien à un vague sens d'identité et d'appartenance, qui doivent plus à la poésie qu'à l'esprit scientifique ${ }^{1}$. Ce n'est que très récemment que nous voyons des tentatives de présenter la chanson traditionnelle à la lumière de données empiriques qui la situent dans un contexte plus large permettant de véritables analyses scientifiques ${ }^{2}$.

1. Voir, par exemple, Germain Lemieux, Chanteurs franco-ontariens et leurs chansons, Sudbury, la Société historique du Nouvel-Ontario, Documents historiques $\mathrm{n}^{\text {os }}$ 44-45, 1963-1964, $113 \mathrm{p}$.

2. Voir la publication récente de Jean-Pierre Pichette, Ah! si l'amour prenait 
Le but de cet article est donc de faire le bilan de la chanson traditionnelle française en Ontario - à la fois du point de vue des recherches et de sa vitalité auprès de ses praticiens - et de tracer son évolution dans le temps et l'espace. Pour ce faire, il faut commencer par déterminer en quoi consiste le répertoire de l'Ontario français : quelles sont ses balises historiques et géographiques (où et quand le répertoire s'est-il implanté sur le territoire), et que comprend-il au juste (combien de chansons, quelles chansons, quelles sortes de chansons). L'article compte aller au-delà de chiffres bruts et des listes de chansons (bien que ceux-ci soient à la base de l'analyse) pour déterminer les caractéristiques du répertoire et pour découvrir comment son rôle et sa fonction ont évolué chez la population qui le maintient.

La seule tentative de présenter un aperçu général du répertoire se dégage de l'inventaire de Jean-Pierre Pichette, Répertoire ethnologique de l'Ontario français, ouvrage qui date déjà de plus d'un quart de siècle ${ }^{3}$. Dans cet inventaire sont identifiées et décrites, selon les normes archivistiques, la plupart des collections importantes recueillies en sol ontarien. On y trouve aussi une liste complète de tous les témoignages de la chanson traditionnelle dans les documents historiques qui précèdent les travaux des folkloristes. Mais à part quelques chiffres bruts, cet inventaire ne nous en dit pas long sur la nature du répertoire. Quelles chansons constituent ces collections ? Quelle est leur distribution sur le territoire ontarien ? Contient-il des variations régionales, a-t-il évolué dans le temps ? Comment se compare-t-il aux répertoires recueillis ailleurs en Amérique française ? Le commentaire de l'auteur qui l'assimile à la tradition québécoise sans en discerner

racine. Chansons populaires du Nouvel-Ontario. Répertoire de Donat Paradis (18921985), cultivateur franco-ontarien, Québec, Les Presses de l'Université Laval, « Les Archives de folklore » 31, 2016, 470 p. Voir aussi l'article de Marcel Bénéteau dans l'Encyclopédie du patrimoine culturel de l'Amérique française : http://www.ameriquefrancaise.org/fr/article-718/Chanson $\% 20$ traditionnelle $\% 20$ française $\% 20 \mathrm{en} \% 20$ Ontario\#.V_05Ma0VDIU (consulté le 15 juin 2017).

3. Jean-Pierre Pichette, Répertoire ethnologique de l'Ontario français. Guide bibliographique et inventaire archivistique du folklore franco-ontarien, Ottawa, Les Presses de l'Université d'Ottawa, 1992, x-230 p. 
de particularités régionales est-il valable pour toute la province, ou seulement pour les régions limitrophes de la mère-province?

J'ai moi-même dû me heurter à ces questions - à l'époque pratiquement impossibles à résoudre - lors de la rédaction de ma thèse de doctorat ${ }^{4}$. Cette étude, qui se voulait une analyse du lien entre la tradition orale et l'identité régionale, employait comme démarche une comparaison des répertoires de deux groupes colonisateurs dans le sud-ouest de l'Ontario afin de déterminer si les différences observées entre les deux pouvaient être reliées aux contextes sociohistoriques particuliers de leur implantation dans la région ; une bonne partie de mes conclusions s'appuyaient sur des données préliminaires - et très générales - concernant le « répertoire laurentien» recueilli dans le nord-est de la province ${ }^{5}$. Une comparaison valable avec la collection ontarienne la mieux connue, celle de Germain Lemieux, n'était pas possible, en raison du fait que seulement une partie de ce corpus avait été recensée pour le Catalogue Laforte ${ }^{6}$, seul outil de recherche disponible à cette époque. Le père Lemieux lui-même, qui m'accueillit chaleureusement en tant que jeune chercheur et qui mit sa collection entière à ma disposition, avait organisé celle-ci selon des principes qui résistaient à toute tentative de comparaison systématique avec d'autres collections. Et, en ce qui concerne les autres collections répertoriées par Pichette - comprenant environ 7500 versions de chansons, selon son recensement - la plupart d'entre elles avaient été constituées après la publication du Catalogue Laforte et n'avaient donc été le sujet d'aucun classement systématique.

4. Marcel Bénéteau, "Aspects de la tradition orale comme marqueurs d'identité culturelle : le vocabulaire et la chanson traditionnelle des francophones du Détroit », thèse de doctorat, Université Laval, 1178 p.

5. Le terme est emprunté à Jean-Pierre Pichette qui s'en sert pour décrire le répertoire diffusé par la diaspora canadienne-française du Québec au XIX ${ }^{\mathrm{e}}$ siècle, d'abord dans son texte "Coup d'œil sur le répertoire traditionnel d'un chanteur franco-ontarien », Cahiers Charlevoix. Études franco-ontariennes 2, Sudbury, Société Charlevoix et Éditions Prise de parole, 1997, p. 159-214, et par la suite par le même auteur dans Ah! si l'amour prenait racine, op. cit., p. 48-49.

6. Conrad Laforte, Le Catalogue de la chanson folklorique française, Nouvelle édition, Québec, Les Presses de l'Université Laval, «Les Archives de folklore » 18-23, 1977-1987, 6 vol. Cet ouvrage sera discuté en profondeur dans les pages qui suivent. 
Pour une part significative des 1700 versions que comporte mon corpus du Sud-Ouest (que j'avais moi-même classées selon les principes du Catalogue), il m'était donc impossible de confirmer que leur distribution s'étendait aux autres régions de l'Ontario; toutes mes observations à cet égard étaient nécessairement nuancées par des réserves comme « selon les sources consultées », " d'après les données du Catalogue Laforte » et « suite à ma conversation avec Germain Lemieux... ». Mes conclusions étaient certainement valables pour la région en question, mais un gros travail d'identification et de classement s'imposait pour quiconque désirait faire une analyse du répertoire de la province entière. La création d'un catalogue de la chanson traditionnelle française en Ontario me semblait incontournable si je voulais poursuivre mes études dans ce domaine.

L'occasion d'entreprendre une telle tâche se présenta lorsque j'obtins le poste de professeur de folklore et ethnologie à l'Université de Sudbury en 2005, ce qui me donna accès à la collection Lemieux (déposée au Centre franco-ontarien de folklore, également à Sudbury) et aussi à l'étonnante collection étudiante des Archives du département de folklore et ethnologie (DFeus) qui, j'allais le découvrir, composait maintenant un corpus de plus de 10000 versions de chansons (dont environ 8000 de tradition orale franco-ontarienne ${ }^{7}$ ). Je décrirai brièvement, dans les pages qui suivent, le processus de classement de ces deux collections ainsi que les étapes supplémentaires dans les efforts de créer un catalogue de la chanson de tradition orale pour tout l'Ontario français. La tâche, qui s'avéra monumentale, occupa mes heures disponibles pendant plus de dix ans : l'identification et le classement de presque 13000 versions de 1409 chansons traditionnelles, recueillies de 1895 à 2010, provenant de toutes les régions de l'Ontario.

7. Lors de la publication du Répertoire ethnologique de Pichette, ce fonds comprenait 2301 versions de chansons réparties en 95 collections (p. 28). Il n'est pas clair si ces chiffres incluaient aussi les chansons littéraires recueillies par les étudiants et les chansons d'informateurs à l'extérieur de la province. 


\section{Quelques définitions}

Avant de procéder, il convient de définir quelques termes et concepts pour le lecteur non initié à la chanson de tradition orale, commençant par l'objet même de l'analyse. Qu'entendons-nous par « chanson de tradition orale » ? La réponse semblerait relever de l'évidence même, mais une définition est difficile à construire. Il y a toujours eu des aspects arbitraires parmi les critères employés par les experts. La notion même est remise en question par l'ethnologue français Yvon Guilcher; ce dernier suggère que la chanson folklorique a été inventée par les collecteurs du XIX ${ }^{\mathrm{e}}$ siècle qui sont ensuite partis à la recherche de ce qu'ils avaient défini ${ }^{8}$. Ce point de vue est sans doute quelque peu caricatural, car Guilcher avoue que l'objet d'étude de ces folkloristes n'est quand même pas un fantasme et il note « le consensus quasi-total avec lequel ils ont isolé une catégorie particulière de chanson populaire ». Résumant le propos de nombreux experts qui ont tenté de définir la chanson traditionnelle, on peut dire que ce consensus admet des chansons qui partagent au moins une pluralité des critères suivants : auteur anonyme, date et lieu de composition indéterminés, transmission par la tradition orale (ou en dehors des réseaux institutionnels), chanson n'ayant aucun texte fixe, mais existant en plusieurs variantes. De plus, la plupart partagent un langage commun que Guilcher caractérise de " prêt-à-porter langagier » composé de mots, d'expressions, de syntagmes, d'incipits et de motifs qui sont transférables d'une chanson à l'autre. Bien que la date et le lieu de composition soient indéterminés (et indéterminables), on peut généraliser pour dire que la plupart des chansons sont d'origine française et qu'elles existaient bel et bien avant l'immigration en Nouvelle-France auX XVII ${ }^{\mathrm{e}}$ et XVIII ${ }^{\mathrm{e}}$ siècles.

Les notions de types et de versions sont aussi essentielles à la discussion qui suit. Comme je viens de l'indiquer, une chanson de tradition orale n'a pas de texte fixe. Les paroles, transmises de

8. Yvon Guilcher, «Les Collecteurs du XIX ${ }^{\mathrm{e}}$ siècle ont-ils inventé la chanson folklorique ? », dans Jean-Loïc Le Quellec (dir.), Collecter la mémoire de l'autre, Niort, Gestes Éditions, collection Modal (Fédération des Associations de Musiques Traditionnelles), 1984, p. 20-31. 
bouche à oreille, sont en constante évolution par l'entremise de chanteurs qui modifient la chanson en oubliant ou en remplaçant des mots, en supprimant ou en ajoutant des vers selon le contexte social, en fusionnant les paroles d'une chanson à celles d'une chanson semblable ou en empruntant le refrain d'une chanson différente ; un processus semblable apporte des modifications à la mélodie. Pour cette raison, les folkloristes considèrent qu'une chanson traditionnelle est la création non seulement d'un auteur inconnu, mais effectivement l'ouvrage d'auteurs multiples, puisque chaque chanteur qui la transmet contribue à sa création. Il s'ensuit qu'aucune de ces performances individuelles n'est supérieure à une autre - chacune est une expression valable qui représente l'état de la chanson à un moment donné et dans un contexte particulier. Les versions ne sont pas des expressions inférieures ou supérieures, dégradées ou améliorées d'un texte original ; contrairement à la poésie écrite, on ne peut jamais saisir le texte original d'une chanson traditionnelle, seulement sa plus ancienne attestation.

On dira donc qu'une chanson peut exister en plusieurs versions. Prenons, par exemple, une chanson bien connue comme Trois beaux canards, qui est représentée par 268 versions dans le répertoire franco-ontarien. Les représentations individuelles peuvent varier selon le texte, le nombre de vers, la mélodie et le refrain («En roulant ma boule » et «V'là l'bon vent » étant les deux refrains les mieux connus pour cette chanson, quoique Marius Barbeau en ait relevé 92 dans une célèbre étude ${ }^{9}$ ). Malgré ces variations, la chanson est toujours reconnaissable d'après son scénario (le fils du roi qui tue un canard blanc avec son fusil d'argent, etc.), sa structure poétique (dans ce cas, vers de huit pieds se terminant toujours par le son « an ») et la persistance de motifs et tours de phrase reconnaissables dans chaque version (fusil d'argent, les yeux qui coulent l'or et l'argent, etc.). Cette idée de chanson « reconnaissable » malgré toutes les variations

9. Marius Barbeau, « Trois beaux canards (92 versions canadiennes) », dans Archives de folklore II, Publication de l'Université Laval, Montréal, Fides, 1947, p. $97-138$. 
possibles résume la deuxième notion à retenir, celle de la chanson type. La chanson type est une abstraction : elle ne représente pas une version originale, complète ou « correcte » de la chanson, mais plutôt un concept qui renferme toutes les possibilités de la chanson et qui permet de rassembler toutes ses versions sous un titre commun.

\section{Identification des chansons}

La première étape vers la construction d'un catalogue de la chanson traditionnelle française en Ontario était donc d'identifier les milliers de représentations particulières (les versions) et de les ranger dans des fichiers d'après leurs types. Ceci permettrait de déterminer la fréquence et la distribution de chaque chanson type et aussi de confirmer si elle était connue sur l'ensemble du territoire, au lieu d'être restreinte à une région particulière. Le chercheur pourrait aussi déterminer si cette distribution était constante dans le temps ou si elle était en croissance ou en décroissance dans le répertoire chanté.

L'identification nécessite l'examen attentif de chaque version recueillie. Le titre donné par les informateurs ne s'avère pas très utile pour cette tâche, étant basé tantôt sur l'incipit («Par un jour en m'y promenant », « C'est dans Paris il y a... », «Écoutez, j'vais vous chanter... », ou un vers ou syntagme prêt à porter qui s'insère facilement dans des douzaines de chansons), tantôt encore sur les refrains (qui peuvent varier d'une version à l'autre ou encore être identiques pour plusieurs chansons différentes) ou parfois même sur une référence personnelle (« La chanson de mon oncle Jos »). Divers chercheurs au fil des ans ont tenté de créer des titres communs pour les chansons de leurs collections, ou du moins de regrouper les versions sous des mots clés, mais ils tiennent rarement compte des efforts de leurs confrères et la prolifération des titres ne fait qu'intensifier le problème.

C'est justement pour régler ce problème que Conrad Laforte développa son Catalogue de la chanson traditionnelle française ${ }^{10}$,

10. Conrad Laforte, op. cit. 
identifiant et classant environ 80000 versions de chansons en provenance des deux côtés de l'Atlantique. Il regroupa ces versions sous 3641 titres, représentant autant de chansons types. Cet ouvrage, largement reconnu comme l'outil de classification incontournable de la chanson traditionnelle pour tous les chercheurs en Amérique française, est à la chanson traditionnelle ce que le catalogue Aarne-Thompson-Uther est au conte populaire ${ }^{11}$. Mais son utilisation n'est pas facile : Laforte ne fournit aucun exemple de chansons et son index de mots clés est beaucoup trop limité. Il inclut toutefois l'information bibliographique pour chaque version qu'il a répertoriée ; lorsqu'il existe une version publiée d'une chanson, elle peut donc en principe être comparée à celle qu'on désire identifier. Mais le plus souvent, il faut la comparer à des versions déposées dans divers fonds d'archives. La difficulté d'accès - tant pour les livres anciens que pour les fonds d'archives dispersés des deux côtés de l'Atlantique - explique pourquoi le présent travail s'est prolongé sur une période de dix ans. Récemment, un autre outil de recherche, utilisé en France et qui s'avère fort utile, est devenu accessible avec la publication du Répertoire de Patrice Coirault ${ }^{12}$. Organisé selon d'autres principes, cet ouvrage a des avantages certains, le principal étant le résumé du scénario qu'il offre pour chaque chanson type. Il fournit aussi les correspondances des titres avec ceux de Laforte, ce qui permet de faire des renvois entre les deux catalogues (on aurait souhaité que les deux chercheurs aient utilisé les mêmes titres pour les mêmes chansons). Mais l'œuvre de Coirault s'appuie sur un corpus plus restreint que celui de Laforte, se limitant aux chansons présentes dans sa collection personnelle (environ 2358 chansons types) et

11. La dernière édition est de Hans-Jörg Uther, The Types of International Folktales. A Classification and Bibliography Based on the System of Antti Aarne and Stith Thompson, Helsinki, Academia Scientiarum Fennica, "FF Communications " 284-286, 2004, 3 vol.

12. Patrice Coirault, Répertoire des chansons françaises de tradition orale, Ouvrage révisé et complété par Georges Delarue, Yvette Fédoroff et Simone Wallon [et Marlène Belly pour le tome III], [Paris], Bibliothèque nationale de France, 1996-2006, 3 vol. Le travail est basé sur le " fichier » de chansons que Coirault développa pour répondre à ses propres besoins de recherche depuis le début du $\mathrm{XX}^{\mathrm{e}}$ siècle jusqu'à sa mort en 1959). 
à leurs versions publiées. Comme une étude complète exige le recours aux deux catalogues, cet article suivra le classement de Laforte, tout en tenant compte des données de Coirault.

\section{Les catégories Laforte}

Contrairement à ses prédécesseurs (incluant Coirault), Laforte jugea les tentatives de classification basées sur les thèmes et les sujets des chansons - c'est-à-dire le contenu des chansons comme étant trop vague et arbitraire; il décida plutôt de fonder son système sur la forme poétique des chansons. Son analyse du vaste corpus ( 80000 versions, rappelons-le, tirées de manuscrits du Moyen Âge, de publications européennes et nord-américaines et de fonds d'archives des deux côtés de l'Atlantique) fit ressortir six formes poétiques stables et bien définies ; chacune possède non seulement ses propres caractéristiques poétiques, mais aussi des thèmes, sujets et fonctions qui lui sont propres et qui reflètent les époques et milieux de composition. Son système de classification tient aussi compte des thèmes, mais il se fonde avant tout sur les six grandes catégories poétiques, que sont :

I. Chansons en laisse

II. Chansons strophiques

III. Chansons en forme de dialogue

IV. Chansons énumératives

V. Chansons brèves

VI. Chansons sur les timbres

Une connaissance pratique des caractéristiques de chaque catégorie servira à bien saisir les propos de l'analyse qui suit.

\section{Chansons en laisse}

La chanson en laisse est de loin la catégorie de chanson traditionnelle la plus populaire chez les chanteurs franco-ontariens aujourd'hui (la situation est identique au Québec). J'examinerai les raisons pour cet engouement un peu plus tard; je m'en tiens, pour le moment, à décrire la forme. La laisse est une forme poétique médiévale qui survit toujours dans le répertoire tradition- 
nel ${ }^{13}$ et qui est bien représentée parmi les chansons les mieux connues en Amérique française : Trois beaux canards, À la claire fontaine, M'en revenant de la jolie Rochelle, La Destinée, la rose au bois, Passant par Paris en vidant les bouteilles sont toutes des chansons en laisse. Leur forme poétique, qui peut sembler relativement complexe à première vue, est des plus simples : la laisse consiste en une série de vers isométriques (même nombre de pieds) et monoassonancés (finissant par le même son ${ }^{14}$ ). On découvre cette structure de base en retirant le refrain et les répétitions qui accompagnent toujours les versions. Pour la chanson Trois beaux canards, déjà évoquée, ce processus dévoile un petit poème composé de vers de huit pieds : «Derrière chez nous $\mathrm{y}$ a-t-un étang/Trois beaux canards s'en vont baignant/Le fils du roi s'en va chassant » etc. Chaque vers se termine par le son « an » (syllabe masculine). On retrouve dans la tradition orale des laisses de six à seize pieds, avec des terminaisons soit masculines ou féminines. Par exemple, « Men revenant de la jolie Rochelle/J'ai rencontré trois jolies demoiselles/J'ai pas choisi mais j'ai pris la plus belle $[\ldots] /$ Je la menai au logis de son père/Quand ell' fut là, elle but à plein verre/ etc. " est une laisse de dix pieds, assonancée en « è » (+ consonne + e muet) (et donc féminine). Je ne m'attarderai pas ici sur toutes les autres règles de prosodie qui gouvernent la forme, mais la plupart, tout comme la base isométrique et monoassonancée, persistent de façon remarquable dans la tradition orale - fait étonnant vu que la majorité de ces chansons sont anciennes de plusieurs siècles.

Et ceci malgré le fait que la forme soit loin d'être statique. Il faut apprécier que la laisse est rarement chantée seule dans la tradition orale : la grande majorité des chansons en laisse sont accompagnées d'un refrain et, comme on a vu, les versions peuvent employer différents refrains. La chanson en laisse fait aussi

13. Pour une description détaillée de la forme dans son contexte historique et actuel, voir Conrad Laforte, Survivances médiévales dans la chanson folklorique. Poétiques de la chanson en laisse, Québec, Les Presses de 1'Université Laval, « Ethnologie de l'Amérique française », 1981, X-300 p.

14. La chanson traditionnelle est fondée sur l'assonance plutôt que la rime exacte. 
libre usage de la répétition. La même laisse se chantera donc selon plusieurs «formules strophiques », comme on appelle la disposition particulière des refrains et des répétitions de vers et même de sections de vers.

Ces caractéristiques expliquent la grande vitalité de cette ancienne forme ainsi que sa popularité au sein de la population francophone. Facile à retenir, grâce aux répétitions et à sa structure monoassonancée, elle est la forme «à répondre » par excellence. La plupart des chansons en laisse possèdent d'ailleurs un rythme entraînant et traitent de thèmes légers, comiques ou satiriques; la plus grande majorité se penche sur les relations hommes-femmes, avant et après le mariage. Laforte divise la catégorie en dix-sept groupes thématiques, de $\mathrm{A}$ à $\mathrm{Q}$; seuls les deux premiers - A Chansons à caractère épique ou tragique et $\mathrm{B}$ Chansons à sujets religieux - s'éloignent de l'ambiance joyeuse générale. Mais j'avance ici que le contenu de la chanson en laisse - les paroles et le scénario - est moins important que sa fonction communautaire ; le vrai message de ces " chansons à répondre » en est un de participation et d'appartenance. Ce qu'on chante est moins important que le fait qu' on chante ensemble. Au Canada, la laisse est d'ailleurs associée aux chants des voyageurs et aux camps de bûcherons ; elle se chante au temps des fêtes et à l'occasion de réunions de famille ou autres temps forts d'expression identitaire. Il ne serait pas faux de dire qu'actuellement, pour la majorité des francophones en Ontario comme au Québec, les termes « chanson traditionnelle » ou « chanson folklorique » se réduisent presque entièrement à la « chanson à répondre »; la plupart de celles-ci sont des chansons en laisse.

Parmi les 355 chansons en laisse types figurant dans le Catalogue international de Conrad Laforte, 220 sont présentes en Ontario français, représentées par 4206 versions (soit 34,2 \% du répertoire provincial).

\section{Chansons strophiques}

Beaucoup plus nombreuses et variées sont les chansons de la 
deuxième catégorie : les chansons strophiques. Laforte en catalogue 866 chansons types, mais le nombre présent dans le répertoire serait facilement le double. Il semble exister un nombre limité de chansons en laisse et c'est cette catégorie qui a fait l'objet des recherches les plus intensives de Laforte ; en conséquence, les laisses qui lui ont échappé sont relativement rares. Par contre, les sources des chansons strophiques semblent presque inépuisables ; tout chercheur qui a fait du terrain dans le domaine des chansons traditionnelles peut montrer un dossier plein de chansons strophiques « non cataloguées » qu'il a recueillies.

La forme des chansons strophiques varie elle-même de façon considérable. Sa caractéristique principale consiste en une série de strophes de longueur fixe $\mathrm{e}^{15}$, au lieu d'être une simple série de vers comme la laisse. La plupart d'entre elles n'ont aucun refrain. Les strophes varient d'un à douze vers, bien que celles de deux à huit vers soient les plus communes dans la tradition orale. Les chansons disposées en distiques et en tercets sont généralement les plus anciennes ; une structure employant plus de huit vers trahit souvent une origine littéraire relativement récente. Les vers ne sont pas nécessairement isométriques; des formes plus complexes apparaissent auX XVII et XVIII ${ }^{\mathrm{e}}$ siècles avec des strophes et des rythmes moins réguliers. Il n'est pas question ici de monoassonance comme dans les chansons en laisse; on trouve des assonances suivies $(a a b b)$ ou croisées $(a b a b)$ dans les chansons à quatrains et une grande variété de rimes croisées et internes dans les strophes plus développées ; les tercets, de leur côté, prennent souvent la forme d'un vers blanc initial (souvent répété de sorte qu'il rime avec lui-même), suivi de deux vers assonancés :

La belle s'y promène dans son jardin de fleur-es Plus blanche que la neige, plus belle que le jour.

Ce sont trois capitaines qui vont lui faire amour.

15. On emploie aussi en français général le mot « couplet » pour « strophe », mais les experts n'utilisent ce terme que pour désigner une strophe de deux vers (distique), tout comme « tercet» pour une strophe de trois vers, « quatrain » pour quatre vers, etc. 
Le plus jeune des trois l'a pris par sa main blanche,

L'a pris par sa main blanche, l'embarque sur son ch'val gris, $\mathrm{Au}$ logis chez son père, pour y passer la nuit.

Etc.

Les thèmes et sujets des chansons strophiques font preuve de beaucoup plus de diversité que ceux qu'on retrouve parmi les chansons en laisse. La plupart sont narratives, présentant des scénarios comiques et tragiques : chansons sur les conditions civiles, départs et retours de soldats et marins, chansons de voyageurs et forestiers, ivrognes, amoureux et couples mariés. On y retrouve aussi des chansons de circonstances chantées à l'occasion de noces et de fêtes religieuses. Ce sont des chansons que les gens écoutent plutôt que des chansons auxquelles ils participent. Il est toujours possible de répéter des vers et de faire chanter l'auditoire, mais en général, ce grand corpus s'élève en parallèle avec la chanson «à répondre » pour remplir une autre fonction : celle de raconter une histoire. On verra dans l'analyse qui suit un déclin prononcé au cours du XX $\mathrm{X}^{\mathrm{e}}$ siècle dans la fréquence et la diversité des chansons narratives dans le répertoire franco-ontarien.

Pour l'Ontario français, 429 chansons strophiques classées par Laforte ont été retenues pour la présente étude, ainsi que 146 chansons non cataloguées mais clairement d'origine traditionnelle, pour un total de 573 chansons types (3 414 versions). Bien que le nombre de chansons strophiques répertoriées en Ontario soit beaucoup plus élevé que pour les chansons en laisse (573 chansons types contre 220), le nombre de versions est pourtant inférieur (3 414 versions ou 27,8\% du répertoire pour les strophiques, à comparer à 4206 versions ou 34,2\% pour les chansons en laisse). L'analyse démontrera que, même si une très grande quantité de chansons strophiques sont présentes dans le répertoire, la plupart ont une distribution limitée et figurent en très peu de versions ; ce sont donc les chansons en laisse qui sont chantées les plus fréquemment. 


\section{Chansons en forme de dialogue}

Les six catégories de Laforte se réduisent effectivement à deux formes principales : on peut dire qu'il y a la chanson en laisse et toutes les autres qui suivent en fait la forme strophique, avec quelques variations. La chanson en forme de dialogue est en tous ses aspects une chanson strophique et peut effectivement être considérée comme une sous-catégorie de cette dernière. Notons que Laforte permet des échanges dialogués à l'intérieur des chansons strophiques de la deuxième catégorie, mais il distingue celles de la troisième catégorie en étant composée entièrement d'un dialogue entre deux ou plusieurs personnages ${ }^{16}$. Les chansons de cette catégorie ne sont pas nombreuses - Laforte n'en classe que 102 types dans son catalogue - et on peut se demander si ce groupe de chansons mérite réellement une catégorie à part entière puisque la plupart d'entre elles pourraient facilement s'intégrer aux thèmes des chansons strophiques. Selon Laforte, la distinction s'impose par « l'originalité et l'ancienneté du répertoire ${ }^{17}$ ». La plupart datent des XVII et XVIII ${ }^{e}$ siècles et reflètent les goûts et les mœurs de cette époque ; notons en particulier les dialogues de bergers et de bergères qui sont souvent des versions populaires et folklorisées de saynètes présentées pour le divertissement des membres de la Cour. Il n'est sans doute guère étonnant que ce genre de chanson ait une place réduite dans le répertoire nord-américain, étant donné les différences d'environnement et de contexte social; encore relativement populaires au début du $\mathrm{Xx}^{\mathrm{e}}$ siècle, ces chansons sont, à toute fin pratique, absentes des collectes les plus récentes en Ontario.

Somme toute, l'analyse relève 77 chansons types en 375 versions, soit $3,1 \%$ du répertoire.

16. Quoique Laforte les définisse comme étant « sans narrateur », il admet toutefois un ou deux vers de mise en scène pour introduire les personnages dans certaines chansons de cette catégorie.

17. Conrad Laforte, Le Catalogue de la chanson folklorique française III. Chansons en forme de dialogue, Québec, Les Presses de l'Université Laval, 1982, p. iii. 


\section{Chansons énumératives}

La chanson énumérative existe dans le seul but d'énumérer quelque chose de façon divertissante : objets, personnes, couleurs, jours de la semaine, qualités ou défauts, chiffres, actions, vêtements ou parties du corps, contenants et contenus, pour ne nommer que quelques possibilités. Elle emploie aussi la forme strophique. Laforte identifie deux sortes d'énumération : l'énumération simple, qui nomme un ou plusieurs nouveaux éléments dans chaque strophe (comme, par exemple, Ah! si mon moine voulait danser) et l'énumération à reprise récapitulative qui, non seulement introduit un nouvel élément à chaque strophe, mais répète à chaque fois tous les éléments introduits antérieurement (Alouette et Savez-vous ce qu'il y a, ou «L'Arbre est dans ses feuilles » étant les exemples les mieux connus de ce genre de chanson que Germain Lemieux qualifie de « chansons cassecou »). La récapitulation fait en sorte que la strophe n'est pas strictement de «longueur fixe», puisqu'elle s'allonge d'un vers à l'introduction de chaque élément.

Sauf quelques exceptions, la fonction narrative est plus ou moins absente des chansons de cette catégorie et plusieurs d'entre elles sont considérées et chantées comme « chansons à répondre ». Elles peuvent servir à des fonctions pédagogiques et de socialisation (énumérant métiers, cris d'animaux, parties du corps, jours de la semaine, préceptes religieux, etc.), mais leur but principal en est un de divertissement. Pour citer Lemieux : « Il va sans dire que les thèmes et musical et poétique de la chanson casse-cou ne sont pas très compliqués. » Et encore : «Nous n'avons pas encore rencontré une chanson casse-cou qui ne fût pas en même temps une chanson à répons. $\rangle^{18}$

Laforte classe 324 chansons énumératives dans le répertoire global, dont 160 sont présentes dans le répertoire ontarien et représentées en 2145 versions (17,4\% du répertoire).

18. Germain Lemieux, op.cit., p. 23-24. 


\section{$V$. Chansons brèves}

Les chansons brèves, comme leur nom l'indique, sont courtes, formées d'une ou de deux strophes seulement, ou parfois même d'un ou de deux vers. Ce sont toutefois des chansons complètes en soi ; il ne s'agit pas de fragments de chansons, mais bel et bien des chansons composées avec la brièveté comme caractéristique principale, car elles remplissent des fonctions spécifiques : berceuses pour endormir, prières ou instructions religieuses, formulettes de jeux, rondes à danser, ralliements de groupes sociaux, chansons attrapes, etc. Cette catégorie pose cependant plusieurs problèmes de classification dont il faut tenir compte dans l'analyse.

En premier lieu, cette vaste catégorie n'a été que partiellement cataloguée par Laforte (elle n'a pas même été considérée par Coirault). Il n'existe donc aucun outil de classification qui tienne compte de la grande diversité de cette catégorie. Laforte avait conçu trois grands groupes de chansons brèves : les chansons que les adultes chantent aux enfants (A Berceuses, $B$ Prières, C Rimettes), les chansons que les enfants chantent entre eux (D Comptines, E Formulettes de jeux chantées, F Rondes à danser) et un vaste répertoire de chansons chantées autant par les adultes que par les enfants (entre autres, chansons de société, aide-mémoire pour airs de violon, cris de marchands ambulants, etc.). Seul l'inventaire des deux premiers groupes - « les enfantines »-a été publié par Laforte. Les chansons brèves « adultes », qui abondent dans la tradition orale, n'ont fait partie d'aucune étude systématique.

En deuxième lieu, l'échantillonnage à partir des collections franco-ontariennes n'est pas fiable. Plusieurs folkloristes semblent n'avoir attaché aucune importance à ce répertoire ; par exemple, Germain Lemieux n'a recueilli qu'une dizaine de chansons brèves parmi les 3000 versions qu'il a recueillies. Plusieurs autres collections n'en tiennent pas compte du tout; par contre, pour d'autres collections, comme celle des étudiants du département de folklore de 1'Université de Sudbury, la catégorie compte pour presque $15 \%$ du répertoire recueilli. Il est donc difficile de déterminer 
leur distribution et leur fréquence sur le terrain. Enfin, il existe un vaste répertoire dont même le plan ambitieux de Laforte ne tient pas en compte : parodies, chansons grivoises et scatologiques, chansons associées au scoutisme et autres mouvements sociaux et au milieu scolaire et portant sur divers sujets hétéroclites ; j'ai donc créé plusieurs nouveaux regroupements pour tenir compte de ces éléments du répertoire.

Laforte identifie 1378 chansons types brèves enfantines dans son Catalogue ; j'ai compté 1296 versions de 235 chansons types de tous les regroupements - enfants et adultes - pour le répertoire ontarien.

\section{Chansons sur les timbres}

Cette catégorie présente aussi quelques problèmes de classification. Laforte y classe des chansons composées sur l'air d'autres chansons ; la définition est en quelque sorte arbitraire, puisque la grande majorité des chansons traditionnelles a évolué de cette façon. L'auteur est parfois connu et les textes sont souvent très littéraires. Très peu de chercheurs ont inclus ce genre de chanson dans leurs enquêtes. La catégorie comprend d'ailleurs plusieurs ordres de chansons : certaines, classées dans le groupe A « Parodies », sont bien établies et acceptées de longue date dans le répertoire traditionnel (Au clair de la lune, Le bon roi Dagobert, Le petit bois d'l'ail). Mais le groupe comprend aussi des parodies plus récentes et ponctuelles. Le groupe B rassemble des chansons historiques dont plusieurs sont également connues depuis longtemps, portant sur des personnages comme le Maréchal Biron et Napoléon en Europe, et des événements comme la Rébellion de 1837 au Bas-Canada. Mais il inclut aussi des chansons qui portent sur des événements plus locaux et ponctuels. Le groupe $\mathrm{C}-\mathrm{et}$ c'est sans doute ici l'apport le plus original de Laforte - offre une classification régionale de chansons locales et comprend des complaintes acadiennes de naufrages, mais aussi des compositions plus légères sur des sujets familiaux et communautaires (noces, personnages locaux, mésaventures comiques, etc.). Le 
Groupe D comprend des chansons politiques et électorales. Enfin le Groupe E rassemble des cantiques religieux basés sur des airs traditionnels.

Cette catégorie manque, à mon avis, de cohérence interne, rassemblant à la fois des chansons composées par des écrivains professionnels et d'autres par des chanteurs anonymes œuvrant véritablement dans la tradition orale. Les chansons locales, en particulier, sont éphémères et ne se transmettent que rarement au-delà du temps et du lieu de leur composition; elles sont donc impossibles à cataloguer systématiquement. Étant donné que très peu de chercheurs ont recueilli des chansons de ce genre, il est difficile de déterminer leur distribution géographique et temporelle. Laforte en recense quand même une quantité impressionnante en Acadie et au Québec (environ 300), mais aucune en Ontario. Les étudiants de l'Université de Sudbury, par contre, enquêtant pour la plupart dans leurs propres familles, relèvent un grand nombre de ces chansons qui commémorent noces, anniversaires et autres expériences personnelles.

J'ai retenu 850 versions de 144 chansons types dans la catégorie des chansons sur les timbres.

Ce détour nécessaire à travers les catégories du Catalogue Laforte aidera à mieux saisir les enjeux de la discussion qui suit, mais aussi à mieux apprécier les formes et les variétés de ce vaste corpus qu'on appelle « chanson de tradition orale »; il aidera aussi à mieux concevoir les fonctions des différentes sortes de chansons. Le Catalogue Laforte est un ouvrage monumental qui s'avère indispensable à toute étude sérieuse de la chanson traditionnelle française. Cependant, son utilité par rapport au répertoire francoontarien est très limitée. Comme je l'ai mentionné plus haut, la plupart des collections franco-ontariennes ont été constituées après la publication du Catalogue ou n'ont tout simplement pas retenu l'attention de son auteur ; la plus importante à l'époque des travaux de Laforte, celle de Germain Lemieux, n'y figure que de façon partielle. Somme toute, Laforte inclut 1248 versions ontariennes dans son Catalogue, soit environ $10 \%$ des chansons 
relevées dans le présent exercice.

Le tableau 1 reprend les quantités et les proportions de chacune des catégories du Catalogue Laforte dans le répertoire franco-ontarien.

\section{Tableau 1}

Fréquence et proportion des catégories Laforte sur l'ensemble du terrain ontarien

\begin{tabular}{|c|c|c|c|c|}
\hline $\begin{array}{c}\text { Catégorie } \\
\text { Laforte }\end{array}$ & Types & Versions & $\begin{array}{c}\text { \% du total } \\
\text { (Types) }\end{array}$ & $\begin{array}{c}\text { \% du total } \\
\text { (Versions) }\end{array}$ \\
\hline I. Laisses & 220 & 4206 & 15,6 & 34,2 \\
\hline II. Strophiques & 573 & 3414 & 40,7 & 27,8 \\
\hline III. Dialogues & 77 & 375 & 5,5 & 3,1 \\
\hline IV. Énumératives & 160 & 2145 & 11,3 & 17,4 \\
\hline V. Brèves & 235 & 1296 & 16,7 & 10,5 \\
\hline VI. Timbres & 144 & 850 & 10,2 & 6,9 \\
\hline Total & $\mathbf{1 4 0 9}$ & $\mathbf{1 2 2 8 6}$ & $\mathbf{1 0 0}$ & $\mathbf{9 9 , 9}$ \\
\hline
\end{tabular}

\section{LE RÉPERTOIRE FRANCO-ONTARIEN}

Pour les besoins de cette analyse, j'ai regroupé les collections selon les trois grandes régions de l'Ontario français : le Nord, l'Est et le Sud. Évidemment, aucune de ces régions ne représente une population ou une culture uniforme et chacune peut être divisée en sous-régions selon des critères géographiques, historiques, économiques et démographiques. Le Sud, par exemple, comprend le Sud-Ouest et le Centre, qui constituent à la fois le plus ancien peuplement francophone de la province et le plus récent ; géographiquement, la Huronie entre aussi dans cette région. Pour le Nord, la région de Sudbury, le Témiscamingue ontarien et la Grande Zone Argileuse ont été peuplés par trois vagues d'immigration distinctes, chacune arrivant dans le cadre d'un contexte socio-économique différent. L'Est, avec sa proximité du Québec, est peut-être plus homogène, mais la division rurale/ urbaine est importante. Malgré ces différences, les répertoires de 
chacune de ces trois grandes régions présentent à l'interne certaines caractéristiques communes qui les distinguent des autres régions.

Du point de vue ethnologique, ces différences historiques sont des facteurs à considérer. La tradition orale est une tradition vivante, en constante évolution, qu'un groupe d'appartenance ne cesse de réévaluer et de recréer. Parmi les éléments du bagage culturel qu'un groupe apporte, ceux qu'il décide de garder, d'éliminer, de modifier dans un nouveau contexte reposent sur des choix collectifs qui découlent des conditions initiales de l'établissement de la communauté, des occupations de ses habitants, des contacts maintenus avec la terre d'origine, des relations avec les communautés avoisinantes, du statut social des premiers immigrants et de l'évolution de ce statut au sein de la nouvelle communauté. Bref, les facteurs historiques, démographiques et économiques sont des facteurs à considérer dans la construction d'un patrimoine oral. Le tableau 2 montre l'apport de chaque région à l'ensemble du répertoire franco-ontarien.

\section{Tableau 2}

Nombre de types et de versions recueillis par région

\begin{tabular}{|l|c|c|}
\hline Région & $\begin{array}{c}\text { Chansons } \\
\text { types }\end{array}$ & $\begin{array}{c}\text { Versions } \\
\text { totales }\end{array}$ \\
\hline Nord & 1060 & 8490 \\
\hline Est & 596 & 1628 \\
\hline Sud & 763 & 2168 \\
\hline Total provincial & $\mathbf{1 4 0 9}{ }^{19}$ & $\mathbf{1 2 2 8 6}$ \\
\hline
\end{tabular}

Il est évident à première vue que la distribution des données sur cet immense terrain n'est pas équilibrée. Le nord de la province - en particulier le Moyen-Nord, rayonnant autour de la ville du Grand Sudbury - a été sillonné beaucoup plus profondément que 1'Est

19. Puisque la même chanson type peut être présente dans plus d'une région, on ne peut additionner la quantité présente dans chaque région pour obtenir un total; le chiffre total représente chaque type compté une fois seulement, qu'il soit connu dans toute la province ou qu'il ne paraisse que dans une seule collection. 
et le Sud et, en conséquence, est surreprésenté dans l'inventaire. Comme nous verrons, ce déséquilibre est dû à l'ampleur des deux collections les plus importantes : celle de Germain Lemieux (1 939 chansons $^{20}$ ) et celle des archives de folklore du département de folklore et ethnologie de 1'Université de Sudbury (Dfeus). Ce dernier fonds, composé de plus de 3000 enquêtes étudiantes déposées depuis 1980, est de loin le plus important dans la province. Il ne se limite pas au Nord ontarien, puisque les étudiants - grâce surtout au programme de cours par correspondance - ont effectué des enquêtes partout en province (et même à l'extérieur). Mais la masse critique demeure centrée sur le Nord : 6153 chansons traditionnelles recueillies sur cette grande région y sont déposées. En ajoutant la collection de Germain Lemieux et les autres collectes faites sur ce terrain, on constate que plus de 8000 chansons - deux tiers du répertoire ontarien - ont été recueillies dans le Nord. L'est de la province est par contre sous-représenté dans les collections par rapport à sa population francophone : 1628 chansons au total (Marius Barbeau et ses collaborateurs et Lucien Ouellet ayant été les collecteurs principaux). Le Sud, pour sa part, qui compte entre autres ma propre collection de 1703 chansons, est très bien représenté par rapport à sa population francophone (2 168 chansons au total).

\section{Analyse des répertoires régionaux}

Dans cette section, je présenterai chacun de ces répertoires régionaux avec une description des collections principales qui les composent, quelques notes sur leur constitution, leurs particularités et l'éclairage qu'ils amènent à la chanson traditionnelle française en Ontario.

L'analyse initiale portera moins sur des chansons individuelles que sur les tendances générales, c'est-à-dire moins sur les chansons particulières que sur les sortes de chansons qui caractérisent

20. La collection Germain Lemieux, déposée au Centre franco-ontarien de folklore à Sudbury, contient en effet 3118 chansons, mais j'ai exclu de ce nombre toutes les chansons recueillies à l'extérieur de la province et toutes celles qui ne répondent pas aux critères acceptés dans la définition d'une chanson de tradition orale. 
chaque répertoire, autant du point de vue formel (forme poétique) que thématique et fonctionnel. Il est à noter que les observations les plus pertinentes portent sur les quatre premières catégories, étant donné le traitement aléatoire des cinquième et sixième catégories par la plupart des chercheurs.

\section{Le Nord}

Le Nord, avec 1060 chansons types représentées par 8490 versions, est la région la plus profondément sillonnée par les enquêteurs et en conséquence la mieux représentée dans l'inventaire du répertoire franco-ontarien. Comme je l'ai mentionné au préalable, cette vaste région comprend trois sous-régions importantes peuplées en autant de mouvements migratoires et faisant chacune preuve de certaines particularités. Un portrait très sommaire voit le début de la colonisation dans la période 1870-1910 pour le Témiscamingue. Traite des fourrures, industrie forestière et agriculture se succèdent pour attirer des colons qui traversent facilement la frontière entre l'Ontario et le Québec ; le développement de mines dans la première décennie du $\mathrm{Xx}^{\mathrm{e}}$ siècle amène de nouveaux groupes de pionniers.

Une autre vague de colonisation s'ouvre avec la construction du chemin de fer Pacifique-Canadien. Le Moyen-Nord accueille de nombreux colons canadiens-français du Québec de 1880 à 1920, établissant une chaîne de paroisses et de communautés de Mattawa jusqu'à Sault-Sainte-Marie. Sudbury devient en quelque sorte la capitale de cette région et développe un réseau institutionnel important. Ici aussi, industrie forestière, agriculture et industrie minière sont à la base de l'économie. Les mines, en particulier, attirent plusieurs autres groupes immigrants qui font de la ville une communauté très cosmopolite. Mais, même si la communauté n'a pas un caractère homogène comme celle que nous verrons dans l'Est ontarien, les francophones peuvent profiter d'une masse suffisante et d'un appui institutionnel pour développer une culture qui s'affirme comme porte-étendard de l'identité francophone. 
Un autre ruban colonisateur s'étire de Timmins à Hearst le long de la Grande Zone Argileuse. Cette région est peuplée de 1910 à 1940 environ. Malgré quelques tentatives d'agriculture au début du siècle, ce sont les mines et la forêt qui prédominent dans l'économie de ces terres au-delà du bouclier canadien ${ }^{21}$.

\section{Les collections}

L'ensemble des collections nous donne une indication de l'évolution du répertoire nord-ontarien, commençant par la collection de François-Joseph Brassard (1945), ensuite celle de Germain Lemieux (1948-1975) et enfin celle des étudiants du département de folklore et ethnologie de l'Université de Sudbury (Dfeus : 1975-2010). La collection de Jean-Pierre Pichette, reçue tardivement au cours de ce projet, s'insère dans le même temps et le lieu que la collection étudiante. Les deux tableaux qui suivent résument la composante de chacune de ces collections, d'après les six catégories du Catalogue Laforte.

\section{Collection François-Joseph-Brassard}

Premier folkloriste à faire du terrain dans le Nord ontarien, François-Joseph Brassard profite de liens de parenté pour effectuer des enquêtes dans cette région de 1943 à 1948. Au cours de ces années, son informateur principal, Urbain Petit, lui chantera 465 chansons ; malheureusement Brassard ne nota que 112 versions de ce répertoire exceptionnel. Urbain Petit, né à Saint-Barthélémy, comté de Berthier en 1872, habita plusieurs endroits au Québec et fit un séjour en Nouvelle-Angleterre avant de s'installer en permanence comme agriculteur à Strickland, près de Cochrane, en 1925, à l'âge de 53 ans $^{22}$. Presque tout son répertoire avait été

21. Pour une description plus détaillée des contextes de peuplement des sousrégions du nord, mais aussi de l'est et du sud de la province, voir Gaétan Gervais, " L'Ontario français (1821-1910) », dans Cornelius J. Jaenen, Les FrancoOntariens, Ottawa, Les Presses de l'Université d'Ottawa, « Ontario Historical Studies Series », 1993, p. 49-124 ; et Gaétan Gervais, «Les Paroisses de l'Ontario français 1767-2000 », Cahiers Charlevoix. Études franco-ontariennes 6, Sudbury, Société Charlevoix et Éditions Prise de parole, 2005, p. 99-194.

22. Pour le portrait de ce chanteur extraordinaire, voir François Brassard, « Re- 
Tableau 3

Nombre total de types et de versions par catégorie dans les collections du Nord ontarien

( $\mathrm{T}=$ types $; \mathrm{V}=$ versions $)$

\begin{tabular}{|c|r|r|r|r|r|r|r|r|}
\hline $\begin{array}{c}\text { Catégorie } \\
\text { Laforte }\end{array}$ & \multicolumn{2}{|c|}{ Brassard } & \multicolumn{2}{|c|}{ Lemieux } & \multicolumn{2}{c|}{ DFEus-No } & \multicolumn{2}{c|}{$\begin{array}{c}\text { Pichette } \\
\text { et autres }\end{array}$} \\
\hline & \multicolumn{1}{|c|}{$\mathrm{T}$} & $\mathrm{V}$ & \multicolumn{1}{c|}{$\mathrm{T}$} & $\mathrm{V}$ & \multicolumn{1}{c|}{$\mathrm{T}$} & $\mathrm{V}$ & $\mathrm{T}$ & $\mathrm{V}$ \\
\hline I. & 26 & 56 & 150 & 719 & 161 & 2287 & 69 & 111 \\
\hline II. & 29 & 30 & 339 & 823 & 209 & 1047 & 55 & 70 \\
\hline III. & 3 & 4 & 51 & 101 & 32 & 100 & 7 & 9 \\
\hline IV. & 16 & 23 & 76 & 216 & 129 & 1373 & 40 & 48 \\
\hline V. & 2 & 2 & 20 & 22 & 119 & 814 & 21 & 23 \\
\hline VI. & 3 & 3 & 35 & 58 & 54 & 533 & 19 & 19 \\
\hline Total & $\mathbf{7 9}$ & $\mathbf{1 1 9}$ & $\mathbf{6 7 1}$ & $\mathbf{1 9 3 8}$ & $\mathbf{7 0 4}$ & $\mathbf{6 1 5 3}$ & $\mathbf{2 1 1}$ & $\mathbf{2 8 0}$ \\
\hline
\end{tabular}

Le tableau 4 transforme ces chiffres bruts en pourcentages pour illustrer la proportion des différentes catégories dans chaque collection :

Tableau 4

Pourcentage des catégories par collection dans le Nord ontarien

\begin{tabular}{|c|r|r|r|r|r|r|r|r|}
\hline $\begin{array}{c}\text { Catégorie } \\
\text { Laforte }\end{array}$ & \multicolumn{2}{|c|}{ Brassard } & \multicolumn{2}{|c|}{ Lemieux } & \multicolumn{2}{c|}{ DFEus-No } & \multicolumn{2}{c|}{$\begin{array}{c}\text { Pichette } \\
\text { et autres }\end{array}$} \\
\hline & \multicolumn{1}{|c|}{$\mathrm{T}$} & \multicolumn{1}{|c|}{$\mathrm{V}$} & \multicolumn{1}{c|}{$\mathrm{T}$} & \multicolumn{1}{c|}{$\mathrm{V}$} & \multicolumn{1}{c|}{$\mathrm{T}$} & $\mathrm{V}$ & $\mathrm{T}$ & $\mathrm{V}$ \\
\hline I. & 32,9 & 47,3 & 22,4 & 37,1 & 22,9 & 37,2 & 32,7 & 39,6 \\
\hline II. & 36,7 & 25,2 & 50,1 & 42,4 & 29,7 & 17,5 & 26,1 & 25,0 \\
\hline III. & 3,8 & 3,4 & 7,6 & 5,2 & 4,5 & 1,6 & 3,3 & 3,2 \\
\hline IV. & 20,3 & 19,3 & 11,3 & 11,1 & 18,3 & 22,3 & 19,0 & 17,1 \\
\hline V. & 2,5 & 1,7 & 3,0 & 1,1 & 16,9 & 13,2 & 9,9 & 8,2 \\
\hline VI. & 3,8 & 2,5 & 5,2 & 3,0 & 7,7 & 8,7 & 9,0 & 6,8 \\
\hline
\end{tabular}


appris dans sa province natale. Brassard indique que monsieur Petit ne se produisait presque jamais en public et chantait rarement même dans des situations familiales. Selon Pichette, on retrouve 134 chansons franco-ontariennes recueillies par Brassard dans les fonds d'archives à l'Université Laval et au Centre canadien d'études sur la culture traditionnelle ${ }^{23} ; 118$ d'entre elles sont confirmées comme étant de tradition orale, d'après le Catalogue Laforte, et sont retenues ici.

Les données de la collection sont trop peu nombreuses pour avancer de grandes hypothèses, mais on peut sans doute considérer le répertoire de monsieur Petit comme étant représentatif des traditions orales amenées par la première génération de francophones à s'établir dans le Nouvel-Ontario, un échantillon d'un patrimoine culturel qui s'implante dans un nouveau territoire. Je note d'ailleurs que les proportions représentées dans la collection établissent le ton et sont tout à fait conformes au modèle qui se dégagera de l'analyse des autres collections du Nord. Comme nous voyons dans le tableau 3, la collection Brassard comprend à peu près le même nombre de chansons en laisse et de chansons strophiques au niveau des types (26 et 29) ; par contre, on voit qu'au niveau des versions, le chiffre total pour les laisses est deux fois plus élevé : 26 types, 56 versions. En même temps, les chiffres pour les chansons strophiques ne bougent guère : 29 types, 30 versions. À une seule exception, les chansons strophiques sont présentes en versions uniques ; en revanche, monsieur Petit connaît en moyenne deux versions de chaque laisse. Les pourcentages éclairent la situation davantage : au niveau des types, les laisses représentent $32,9 \%$ du répertoire et les chansons strophiques $36,7 \%$. Mais dans la colonne des versions - c'est-à-dire le total des chansons chantées - les proportions sont complètement renversées : en fait, à 47,3\%, les laisses constituent presque la moitié de la collection, tandis que la proportion des chansons

cordeur de chansons », dans Archives de folklore, Montréal, Éditions Fides, vol. 2, « Hommage à Marius Barbeau », 1947, p. 191-202.

23. Pichette, Répertoire ethnologique, op. cit., p. 163. 
strophiques chute à un quart $(25,2 \%)$ du répertoire. L'autre catégorie importante de cette collection est celle des chansons énumératives $(19,7 \%)$, considérées, comme les chansons en laisse, des « chansons à répondre ». Si on combine les pourcentages de ces deux catégories, on constate que ce genre de chanson forme plus de deux tiers du répertoire chanté par Urbain Petit (67\%).

J'avance ici la notion que les types et les versions éclairent chacun un aspect différent du répertoire, observation qui deviendra plus évidente au cours de l'analyse : les types reflètent la diversité du répertoire et sont une mesure de sa richesse ; les versions, pour leur part, représentent l'expression réelle du fonds en question.

\section{Collection Germain-Lemieux}

Le père Germain Lemieux est le grand folkloriste de l'Ontario français. On considère, à juste titre, les 33 volumes de la série Les Vieux m'ont conté, mettant en vedette les 646 contes et légendes qu'il a recueillis, un des monuments du patrimoine francoontarien. Beaucoup moins connu, son travail sur la chanson traditionnelle constitue une collection de 3118 chansons recueillies entre 1948 et 1975 . Ses enquêtes ne se sont pas limitées à l'Ontario : environ un tiers des chansons provient du Québec - notamment de la Gaspésie, sa région natale - et quelques autres ont été recueillies au Manitoba et même en France. La récolte ontarienne se chiffre à 1939 chansons, collectées principalement dans la région du Moyen-Nord, c'est-à-dire le corridor de la route transcanadienne de Mattawa à Sault-Sainte Marie, avec une concentration majeure dans la région du grand Sudbury. Les enregistrements sonores, ainsi que les transcriptions, avec notation musicale, sont déposés au Centre franco-ontarien de folklore, institution que le père Lemieux mit sur pied pour assurer la sauvegarde de ses travaux et la continuation de ses recherches. Outre deux petits cahiers publiés par la Société historique du Nouvel-Ontario en 1949 et 1950 et deux autres qui paraissent en 1974 et 1975 - un total de 154 chansons - la collection demeure 
complètement inédite ${ }^{24}$.

Comme je l'ai mentionné ci-dessus, Le Catalogue Laforte ne tient compte de la collection Lemieux que de façon parcellaire : seulement 781 de ces chansons franco-ontariennes - soit $40 \%$ du total recueilli - y figurent ${ }^{25}$. L'une des premières étapes que j'ai entreprises fut donc de compléter la classification de la collection de Germain Lemieux, travail qui avait été commencé par Lucie Beaupré à l'Université de Sudbury et que j'ai mené à terme lors de mon entrée en poste au département de folklore de l'Université de Sudbury. La collection complète comprenait, en plus des chansons recueillies à l'extérieur de l'Ontario, bon nombre de chansons de sources littéraires ; j'ai enfin retenu 1939 versions de 671 chansons types pour l'analyse qui suit.

Les chiffres du tableau 3 montrent que les chansons en laisse et les chansons strophiques prédominent ici aussi et on note, comme chez Brassard, une tendance qui va se maintenir dans la plupart des régions de l'Ontario français : bien que les chansons strophiques soient plus nombreuses au niveau des chansons types (150 laisses, 339 chansons strophiques), elles sont chantées bien moins souvent que les chansons en laisse : chaque chanson en laisse est représentée en moyenne par cinq versions, tandis que les chan-

24. Germain Lemieux, Folklore franco-ontarien : chansons I. Sudbury, Société historique du Nouvel-Ontario [désormais SHNo], « Documents historiques » 17, 1949, 48 p. ; Folklore franco-ontarien : chansons II. Sudbury, SHNo, « Documents historiques » 20, 1950, 48 p. ; Chansonnier franco-ontarien I. Sudbury, SHNo, « Documents historiques » 64, 1974, 138 p. et Chansonnier franco-ontarien II. Sudbury, SHNO, « Documents historiques » 66, 1975, 135 p.

25. Selon certaines sources, des différences irréconciliables concernant la méthode de classification des chansons auraient mis une fin prématurée à la collaboration entre Lemieux et Laforte. Dans l'article publié dans les actes du colloque « L'Euvre de Germain Lemieux ", Conrad Laforte parle d'un séjour d'une semaine à Sudbury pour évaluer le travail à faire pour inclure la collection Lemieux dans son Catalogue. Selon lui, "Nous en avons alors inventorié 2576 chansons de tradition orale. Il en restait donc 542 à identifier, en partie des chansons de forestiers et d'histoire locale. » Il offre, sans explication, le commentaire suivant : " Mais comme le résultat de ce dernier inventaire est encore inédit, nous devons nous en tenir [à l'échantillon de 800 chansons que Lemieux avait fourni à Luc Lacourcière] ». Conrad Laforte, " Caractéristiques des chansons du répertoire de Germain Lemieux », dans Jean-Pierre Pichette (dir.), L'Euvre de Germain Lemieux, s.j. Bilan de l'ethnologie en Ontario français. Actes du colloque tenu à l'Université de Sudbury les 31 octobre, $1^{\text {er }}$ et 2 novembre 1991, Sudbury, Centre franco-ontarien de folklore et Prise de Parole, 1993, p. 293. 
sons strophiques, deux fois plus nombreuses, sont représentées en moyenne par 2,5 versions chacune. Lemieux reflète d'ailleurs cette préférence populaire pour les chansons «à répondre »dans le choix des chansons publiées dans ses cahiers chansonniers : bien qu'il ait recueilli deux fois plus de chansons types dans la catégorie strophique, ce sont les laisses qui prédominent, comptant pour deux tiers du répertoire publié.

Mais, en fait, le tableau 4 illustre que la proportion de chansons strophiques demeure très élevée : 50,1\% des chansons types et $42,4 \%$ des versions chantées, ce qui confirme la grande richesse de cette collection. Les chansons narratives (strophiques et dialoguées) et les chansons « à répondre » (laisses et énumératives) sont représentées presque en proportions égales qui peuvent toutes les deux être arrondies à $48 \%$ du répertoire. Les deux autres catégories - chansons brèves et chansons sur les timbres - sont représentées en proportions négligeables; cette pénurie témoigne plutôt des intérêts du père Lemieux que de l'absence de ces chansons dans les répertoires de ses informateurs. Comme tous les grands folkloristes avant lui, Lemieux veut illustrer la diversité de formes et de refrains que peut avoir la laisse des Trois beaux canards (57 versions) ; aussi semble-t-il avoir un engouement spécial pour Le petit mari (44 versions), laisse bien connue qui pourtant ne figure nulle part ailleurs avec une fréquence aussi élevée. Mais il démontre aussi un penchant particulier pour les longues chansons narratives - et pourquoi pas, puisque le chercheur a œuvré avec tant d'assiduité dans le domaine du conte - et sa collection compte plus de chansons à caractère épique et tragique que toute autre collection en Ontario. Les chansons de retour du soldat représentent un autre thème pour lequel il semble éprouver une affection particulière, chansons qui « constituent un lien qui nous rattache à cette lointaine noblesse guerrière médiévale dont le lot était de s'illustrer à la guerre ou d'y laisser la vie ${ }^{26} \gg$. Les chansons en forme de dialogue - surtout les thèmes de bergers et bergères et de jeunes filles à marier et leurs

26. Lemieux, Chanteurs franco-ontariens..., op. cit., p. 36. 
mères - sont aussi très bien représentées. Il enrichit le répertoire traditionnel global avec 62 chansons qui ne semblent avoir été recueillies nulle part ailleurs dans la francophonie. Conrad Laforte lui-même caractérise le répertoire comme l'un « des plus variés, des plus riches et des plus beaux ${ }^{27} \gg$.

La valeur que Germain Lemieux attribue au folklore francoontarien mérite quelques commentaires ici et est pertinente à l'analyse globale du répertoire. Dans Chanteurs franco-ontariens et leurs chansons, Lemieux vante la richesse de la région de Sudbury pour le chercheur en folklore : « Le seul groupe canadienfrançais de la région de Sudbury forme comme un résumé des nombreux centres traditionnalistes du Québec ou de 1'Acadie ${ }^{28}$. » Dans une note en bas de page, il élabore :

M. Théodule Miville était gaspésien de naissance; M. Adélard Boulay venait de la région de Rimouski ; M. Camille Chiasson, qui nous a conservé des dizaines de contes acadiens, était originaire du Nouveau-Brunswick; les familles Bujold de Coniston, où nous avons trouvé d'excellents danseurs et chanteurs, viennent de la Baie-des-Chaleurs ; les Pelland de Sudbury, chanteurs inépuisables, sont originaires de la région de Joliette ; M. Aldéric Perrault, conteur et chanteur réputé, est natif de Saint-Théodore-de-Chertsey, comté de Montcalm ; M. Joseph Dufresne venait de Saint-Félix-de-Valois ; Mme Arthur Parent (Anna Bélanger) a passé une grande partie de sa vie près de Rimouski ${ }^{29}$.

Comme la collection de Brassard - mais de façon beaucoup plus convaincante en raison de son envergure - celle de Lemieux témoigne de l'implantation d'un patrimoine oral sur un nouveau terrain par la première génération - ou tout au plus, la deuxième à s'y établir. Il n'est pas étonnant que certains chercheurs, s'appuyant principalement sur cette collection, aient vu une absence de particularités régionales dans cette extension naturelle du

27. Conrad Laforte, « Caractéristiques des chansons du répertoire de Germain Lemieux », op. cit., p. 302.

28. Lemieux, ibid., p. 14.

29. Lemieux, loc. cit. 
répertoire québécois. Cependant, la valeur réelle de la collection de Germain Lemieux est qu'elle sert d'étalon pour évaluer les autres collectes qui ont été effectuées en Ontario. Sa pertinence ressort en particulier lorsqu'on la juxtapose avec la suivante, celle des étudiants du département de folklore et ethnologie de l’Université de Sudbury.

\section{La collection DFEUS-NO ${ }^{30}$}

Les Archives du département de folklore et ethnologie de 1'Université de Sudbury, établies par Jean-Pierre Pichette, représentent un des fonds d'archives les plus importants dans toute la province; en ce qui concerne le patrimoine immatériel des francophones de 1'Ontario ; ce fonds est sans égal et se compare favorablement aux Archives de folklore de l'Université Laval, au Centre d'études acadiennes à Moncton et aux archives de folklore de 1'Université Memorial à Terre-Neuve ${ }^{31}$. Dès la fondation du département en 1981, les étudiants sont interpelés pour faire des enquêtes de terrain comme composante pratique de leurs cours et leurs travaux sont déposés dans un fonds d'archives. Les sujets d'enquêtes sont très variés et touchent, comme le programme d'études, aux trois domaines classiques du folklore : la culture matérielle, la culture immatérielle et la vie sociale. Les enquêtes portent sur la tradition orale, bien sûr - contes, légendes, chansons, croyances et superstitions, proverbes, dictons et autres formes brèves - mais, avec l'introduction de nouveaux cours au fil des ans, les sujets se sont multipliés pour inclure coutumes et traditions des fêtes populaires et de la vie privée (les rites de passage), médecine populaire, cuisine traditionnelle, religion populaire,

30. Pour les besoins de l'analyse, j'ai divisé la collection des étudiants du département de folklore et ethnologie selon les grandes régions francophones de la province. Dfeus-No comprend les données recueillies dans le Nord ontarien et Dfeus-eo dans l'Est ; le Sud sera sous-divisé en le Sud-Ouest (Dfeus-so) et le Centre (DFeus-CE).

31. Pour l'histoire détaillée de cette collection, veuillez consulter le Répertoire ethnologique de Jean-Pierre Pichette (op.cit.) qui a conçu et orienté ce fonds ; l'organisation et l'uniformisation des collections avec fiches d'enquêtes et inventaires ainsi que les divers dénombrements effectués après 1988 se sont avérés indispensables pour la compilation des données incluses dans la présente étude. 
métiers traditionnels. L'introduction d'un programme complet à distance en 1995 multiplie le nombre d'étudiants et élargit le terrain d'enquête à toute la province. Aujourd'hui, les archives contiennent plus de 3000 enquêtes dont chacune comprend des entrevues enregistrées avec un ou plusieurs informateurs ainsi que leurs transcriptions ou rapports de synthèse.

Mais le département a longtemps manqué de ressources et de personnel pour traiter de façon adéquate ce fonds extraordinaire. Malgré les intentions de mon prédécesseur, lors de mon arrivée à l'Université en 2005, j'ai constaté que les collections avaient été remisées pêle-mêle dans des boîtes dans un coin du sous-sol. J'ai dû dépenser des énergies considérables afin de convaincre l'administration de la valeur de ces collections ; éventuellement un local fut consacré aux archives et suite à plusieurs projets d'été avec l'aide d'étudiants le fonds entier fut classé et organisé par collection. Une banque de données électroniques fut créée permettant une recherche par collecteur, informateur, date et lieu de la collecte et sujet de l'enquête. Environ deux tiers des enregistrements ont été numérisés (la plupart étant sur cassettes) et le contenu de presque 2000 enquêtes a été analysé et entré dans la banque de données. Le travail demeure inachevé en attendant une décision sur l'avenir du département.

Une attention particulière fut portée aux chansons recueillies : toutes les chansons traditionnelles collectées avant 2013 ont été identifiées et les paroles de plus de 10000 versions transcrites remplissent un classeur complet organisé selon les catégories du Catalogue Laforte. De ce total, environ 2000 chansons ont été recueillies à l'extérieur de l'Ontario, laissant plus de 8000 versions franco-ontariennes. Comme indiqué, la grande majorité de celles-ci - plus de 6000 - proviennent du nord de l'Ontario, le bassin principal de recrutement de l'Université de Sudbury.

Cette collection est notable à plusieurs égards. En plus de son échantillonnage massif - qui lui donne en soi un poids considérable - les conditions de la collecte sont très différentes de celles menées par des folkloristes de carrière. La plupart des 
étudiants ont effectué leurs enquêtes auprès de leurs proches : parents, grands-parents, amis et voisins, ce qui donne aux entrevues une qualité de familiarité et d'intimité qu'un chercheur professionnel n'atteint que rarement avec ses informateurs. Si dans quelques familles les résultats sont colorés par une certaine pudeur, dans bien d'autres instances les filtres en place dans une enquête formelle sont complètement absents : chansons grivoises et scatologiques abondent dans certaines collections; on y retrouve aussi une richesse de chansons brèves et de chansons de composition locale qui seraient rarement chantées en dehors de la famille.

Un fonds d'archives ainsi constitué comporte inévitablement certaines faiblesses. Ces enquêtes étant composées de travaux d'étudiant, leur qualité varie considérablement. Toutefois, le manque de diligence des uns est compensé par la débrouillardise et l'originalité des autres. Certes, les chansons les plus populaires sont peut-être surreprésentées ; même l'étudiant le plus assidu ne refusera pas une chanson « trop bien connue » pour atteindre au minimum de pièces exigées par le professeur. Mais, par la même mesure, il acceptera aussi des pièces que peu d'informateurs n'oseraient chanter à un membre de l'élite religieuse ou universitaire. Bref, compte tenu de l'ampleur de l'échantillonnage et de la façon ouverte dans laquelle elle a été constituée, la collection étudiante est sûrement la plus inclusive et, il me semble, celle qui reflète le plus fidèlement ce que les gens chantent vraiment.

Sur le plan diachronique, la collection ouvre une fenêtre très importante. Le territoire couvert par la collection DFEUs-NO correspond en grande mesure à celui parcouru par Germain Lemieux et la collecte commence exactement au moment où ce dernier mettait fin à ses enquêtes de terrain. Les enquêtes de Lemieux couvrent la période de 1948 à 1975 ; les premières enquêtes étudiantes débutent même avant la fondation du département en 1981 et se poursuivent au-delà de 2010. Nous avons donc un recensement ininterrompu du répertoire nord-ontarien sur une durée d'environ 65 ans, qui commence avec les chansons amenées par les membres 
de la première génération à s'établir dans le Nord ontarien et qui finit avec ce que chantent leurs arrière-petits-enfants dans la première décennie du XXI ${ }^{\mathrm{e}}$ siècle. Ces données permettent d'observer sous plusieurs angles l'évolution de la chanson traditionnelle sur ce terrain fertile et offrent de nouvelles perspectives sur la nature et le rôle de la tradition orale face aux changements dans la société.

On voit dans le tableau 3 que les étudiants ont recueilli 704 chansons types représentées par 6153 versions. Il est intéressant de noter que, bien que le total de versions recueillies soit trois fois plus grand que le total de Lemieux (1 938 contre 6 153), le nombre de chansons types n'a pas augmenté de façon significative (671 contre 704); en d'autres mots, on constate que le corpus de 2000 versions et celui de 6000 versions ont tous les deux comme point de départ un fonds d'environ 700 chansons types. La plus grande collection nous fournit certainement beaucoup plus de versions de chaque chanson, mais guère plus de chansons différentes. Évidemment, ce ne sont pas nécessairement toutes les mêmes chansons types dans chaque collection mais les résultats suggèrent que la diversité des chansons sur un terrain n'est pas illimitée.

Les deux collections sont presque identiques en ce qui a trait aux chansons en laisse : Lemieux répertorie 150 chansons types différentes en forme de laisse, tandis que la collection étudiante relève 161 chansons types. Au niveau des pourcentages, il n'y a aucune différence significative : la laisse représente $22,4 \%$ des chansons types recueillies par Lemieux et 22,9\% de celles recueillies par les étudiants. Ces proportions se maintiennent pour les versions chantées : $37,1 \%$ et $37,2 \%$ respectivement. Mais le portrait s'avère tout à fait différent quand on compare les proportions de chansons strophiques : la moitié des chansons types recueillies par Lemieux sont strophiques tandis que moins d'un tiers de la collection étudiante est composé de chansons strophiques $(50,1 \%$ contre $29,7 \%$ ). En d'autres mots, $20 \%$ - ou une sur cinq - des chansons types strophiques disparaissent du répertoire au cours de la période 1975 à 2010. Les proportions frappent davantage au niveau des versions : pour Lemieux, les versions de chansons 
strophiques composent encore $42,3 \%$ du répertoire chanté mais cette catégorie chute à $17,5 \%$ du total des versions recueillies par les étudiants. La chanson strophique s'avère donc un choix beaucoup moins fréquent avec le passage du temps. Puisque les proportions de chansons en laisse demeurent constantes, il y a lieu de se demander ce qui remplace les chansons strophiques.

Ce ne sont certainement pas les chansons en forme de dialogue, l'autre catégorie qu'on peut considérer strictement narrative. Jamais nombreuses, elles baissent de 5,2\% du répertoire dans la collection Lemieux à la proportion minuscule de 1,6\% chez les étudiants, soit cent versions parmi les 6154 recueillies. Trente-cinq de ces versions ont été collectées avant 1990, mais cette tranche du répertoire comprend presque la totalité des chansons des groupes A « La belle et l'amant » et $\mathrm{B}$ « La bergère et le galant» recueillies par les étudiants. Le groupe $\mathrm{C}$ « La fille et la mère (ou le père) » retient une certaine popularité dans les décennies subséquentes, mais la plus grande partie des chansons en dialogue - presque toutes recensées après 1990 - tombe dans le groupe $\mathrm{H}$ « Une personne et un groupe », représenté par des chansons bien connues comme $D$ 'où viens-tu, bergère (19 versions) et des chansons associées à des jeux ou des danses telles $J$ 'a un beau château (14 versions) et La Bastringue (n.c. ${ }^{32}$ ) (12 versions). Ce sont des chansons dans lesquelles la fonction narrative est tout à fait absente; qui plus est, elles sont souvent reliées au contexte d'activités scolaires et recueillies principalement auprès d'enseignants et d'enfants. Si on ajoute une autre chanson bien représentée, Frère Siméon (n.c.) (12 versions), qui semble être de tradition orale malgré sa diffusion récente par divers médias ${ }^{33}$, on discerne une autre tendance qui commence à s'affermir, à savoir que le système scolaire et les sources médiatiques prennent davantage d'importance dans la transmission du folklore. Malgré ces

32. n.c. - non cataloguée, c'est-à-dire absente du Catalogue Laforte.

33. Chanson grivoise popularisée par l'artiste québécois Nono Deslauriers ; la chanson est en fait traditionnelle, selon Patrice Coirault, qui la classe sous le titre Frère Jacques et la petite Guillaumette (11909). Elle ne figure pas au Catalogue Laforte. 
quelques témoignages indiquant que la forme n'est pas tout à fait éteinte, la quantité minuscule des chansons en forme de dialogue suggère que ces dernières sont, à toute fin pratique, absentes du répertoire contemporain dans le nord de l'Ontario.

En comparaison, les trois prochaines catégories de chansons sont en voie d'expansion rapide. Les chansons énumératives figurent deux fois plus fréquemment dans le répertoire étudiant que dans celui de Lemieux (22\% contre $11 \%$ ). Pourtant, le père Lemieux lui-même était exceptionnellement friand de ce genre de chansons - on n'a qu'à lire son éloge de la « chanson casse-cou » (chanson à reprise récapitulative) dans Chanteurs franco-ontariens et leurs chansons ${ }^{34}$ pour apprécier à quel point il considère cet élément du répertoire comme étant presque emblématique des Canadiens français. Conrad Laforte, pour sa part, souligne l'importance de ce volet dans la collection Lemieux et la rareté d'une chanson énumérative (Beau Maréchal) que Lemieux aurait recueilli trois fois dans le Nord ontarien (les seules versions connues en Amérique du Nord) ${ }^{35}$. Mais, contrairement aux étudiants, le père Lemieux n'avait aucun intérêt pour certaines chansons extrêmement bien connues. Par exemple, sa collection ne compte aucune version d'Alouette, gentille alouette, de $\mathrm{Si}$ mon moine voulait danser ou de Bonhomme sais-tu jouer qui comptent, respectivement, 61, 102 et 81versions - soit un total de 244 versions dans la collection étudiante. Aussi compila-t-il sa collection avant que Savez-vous ce qu'il y a (popularisé sous le titre «L'arbre est dans ses feuilles ») ne devienne un succès médiatique (77 versions étudiantes). Mais la nature de la chanson énumérative ressemble à celle de la chanson en laisse : chanson entraînante et amusante, pour qui la fonction narrative est de loin supplantée par la fonction communautaire. La popularité croissante des chansons énumératives dont témoigne amplement la collection étudiante indique donc une tendance de croissance des chansons à répondre aux dépens des chansons narratives.

34. Lemieux, Chanteurs franco-ontariens..., op. cit., p. 22-25.

35. Laforte, "Caractéristiques... », op. cit., p. 296. 
Germain Lemieux ne montra pratiquement aucun intérêt pour les chansons brèves ; les étudiants de folklore, par contre, en ont recueilli plus de 800 dans le Nord ontarien (13,2\% du répertoire). Il faut sans doute tenir compte du fait que la transcription d'une chanson brève demande beaucoup moins de travail qu'une pièce plus longue; mais plus important est le fait que, puisque les étudiants font souvent des enquêtes auprès de leurs parents ou grands-parents, il semble tout à fait normal de retrouver dans leurs travaux une plus grande proportion de chansons enfantines - berceuses et rimettes enfantines, formulettes de jeux chantées pour lesquelles ils maintiennent sans doute des souvenirs d'enfance. Et, en renonçant à la tendance des folkloristes d'enquêter auprès des plus vieilles personnes que l'on puisse trouver, plusieurs étudiants ont aussi fondé leurs recherches sur leurs petits frères et petites sœurs ; les enfants, en général, ont un penchant pour les chansons brèves - parodies et chansons comiques de toutes sortes. D'autres encore ont construit leurs enquêtes autour de leur cercle d'amis ou colocataires en résidence universitaires (ici les chansonnettes grivoises et scatologiques foisonnent). Nous devons donc à cette collection une appréciation de la place réelle des chansons brèves dans le répertoire francoontarien.

Les chansons sur les timbres sont relativement bien représentées dans la collection étudiante $(8,7 \%)$ du répertoire, incluant les parodies bien connues comme Au clair de la lune (68 versions), Le petit bois d'l'ail (« Youpe, youpe sur la rivière », 42 versions) et la chanson historique Un Canadien errant (22 versions). On y retrouve aussi des chansons macaronis ${ }^{36}$ comme I went yesterday sur le bord des États (16 versions) et Prendre un verre de bière - right through (49 versions), qui ne figurent pas au Catalogue Laforte malgré leur grande diffusion dans la société contemporaine, en plus d'une centaine de chansons non identifiées aux sujets variés. C'est sans doute la rubrique $\mathrm{C}$

36. Chansons bilingues comprenant un mélange paroles et syntagmes en français et en anglais. 
«Chansons locales » qui offre le plus grand intérêt ici. Composées au sujet d'événements locaux et ponctuels, la plupart ne survivent pas longtemps et ne voyagent pas loin de leur lieu d'origine. Mais comme je l'ai observé ailleurs, ce genre de chansons est au cœur même du processus folklorique et représente l'interaction de la communauté avec la tradition, permettant à cette dernière de commémorer des événements vécus en les inscrivant dans la conscience collective au moyen d'un modèle narratif et musical bien connu ${ }^{37}$. La collection étudiante comprend 74 chansons qui se placent sous cette rubrique, chansons composées pour commémorer des célébrations familiales comme des mariages, des anniversaires, des départs à la retraite, et autres. C'est la seule collection à ma connaissance qui inclut ce genre de chansons en si grande proportion; cet échantillonnage important représente un autre volet original du répertoire étudiant qui mérite à lui seul une étude approfondie.

Comment résumer enfin les caractéristiques et l'apport de cette collection touffue ? Numériquement, géographiquement et chronologiquement, elle s'impose comme la plus importante en Ontario et l'envergure de l'échantillonnage compense de loin les déficiences méthodologiques de certains enquêteurs qui l'ont constituée. Elle peut être considérée comme la continuation de l'œuvre de Germain Lemieux et illustre de façon très claire l'évolution du répertoire du Nord ontarien depuis son importation par les premiers colonisateurs. Le répertoire signale aussi l'importance croissante du système scolaire et des médias dans la transmission du répertoire traditionnel ; il fait aussi ressortir l'appauvrissement de ce répertoire à la fois par le nombre de chansons de certaines catégories et par la standardisation des versions recueillies. On y voit une évolution nette en faveur des « chansons à répondre » (laisses et chansons énumératives). Un

37. Voir Marcel Bénéteau, «L'Art perdu de "faire des chansons" : la chanson de composition locale dans la région du Détroit», dans Cahiers Charlevoix. Études franco-ontariennes 10, Ottawa, Société Charlevoix et Les Presses de l'Université d'Ottawa, 2014, p. 255-314, pour les caractéristiques de cette forme de chansons et une discussion de ses relations problématiques avec plusieurs chercheurs. 
coup d'œil sur les dix chansons les plus populaires du répertoire illustre bien ce point :

$\begin{array}{ll}\text { I G-10 } & \text { A la claire fontaine (189 versions) } \\ \text { I. B-7 } & \text { Trois beaux canards (148) } \\ \text { II. R-6 } & \text { Chevaliers de la table ronde (137) } \\ \text { I. M-6 } & \text { La Destinée, la rose au bois (102) } \\ \text { IV. Ea-3 } & \text { Ah! si mon moine voulait danser (102) } \\ \text { I. I-8 } & \text { La Fille et la caille (86) } \\ \text { I. K-4 } & \text { M'en revenant de la jolie Rochelle (85) } \\ \text { IV. Mb-1 } & \text { Bonhomme, sais-tu jouer ? (81) } \\ \text { IV. Ea-14 } & \text { Pour boire il faut vendre (79) } \\ \text { IV. Kb-3 } & \begin{array}{l}\text { Savez-vous ce qu'il y a ? } \\ \end{array} \\ & \text { (L'arbre est dans ses feuilles) (77) }\end{array}$

La moitié de ce palmarès est composé de chansons en laisse (I) et quatre autres sont des chansons énumératives (IV); elles tombent assurément toutes sous le concept de « chansons à répondre ». Une seule chanson strophique (II) y figure : Chevaliers de la table ronde est classée comme telle simplement parce qu'elle ne répond pas au critère de la monoassonance; à toute fin pratique, elle se chante aussi comme une « chanson à répondre ».

\section{Jean-Pierre Pichette et autres}

Enfin, 280 versions de chansons tirées principalement de la collection de Jean-Pierre Pichette viennent compléter les données du répertoire du Nord ontarien. Dans son analyse du répertoire de Donat Paradis ${ }^{38}$, Jean-Pierre Pichette relève une typologie qui est tout à fait conforme aux proportions que nous avons relevées jusqu'à présent : $38 \%$ pour les chansons en laisse et $22 \%$ pour les chansons énumératives (dont $60 \%$ de chansons « à répondre ») et seulement $25 \%$ pour les chansons strophiques (les autres catégories étant présentes en proportions minimes). Il observe d'ailleurs que $79 \%$ du répertoire est composé de chansons « gaies et entraînantes » et conclut : « En ce sens, le répertoire Paradis

38. Pichette, Ah! si l'amour prenait racine, op. cit., p. 48-49. Les pourcentages cités ci-dessus sont de cet ouvrage et ne changent pas de façon significative avec l'inclusion d'autres sources dans la dernière colonne du tableau 4. 
se révèle tout à fait conforme au modèle "laurentien" du Québec qui est son foyer d'origine ${ }^{39} \gg$. Rien de cela ne va à l'encontre des tendances suggérées par les autres collections du Nord ontarien.

\section{L'Est ontarien}

L'est de la province nous fournit un répertoire de 596 chansons types représentées par 1628 versions. Le territoire comprend principalement le comté de Prescott-Russell et la ville d'Ottawa, s'étendant jusqu'à Cornwall au sud et Pembroke à l'ouest. Le peuplement francophone de la rive sud de la rivière Outaouais commence vers 1840, précédant ainsi la colonisation du Nord. Le développement de l'industrie forestière attire la main-d'œuvre du Bas-Canada ; les terres ainsi ouvertes accueillent par la suite des agriculteurs qui fondent plusieurs communautés entre Ottawa et Cornwall. Bien plus que les autres régions de l'Ontario, ce territoire fait partie de l'expansion naturelle de la population québécoise ; les liens sociaux et culturels avec la province d'origine sont donc bien plus faciles à maintenir qu'ils le sont dans les régions plus éloignées. Le choix d'Ottawa comme capitale nationale aura un effet marquant sur le développement de cette francophonie. Comme Gaétan Gervais l'explique : «Renforcé par la proximité de Québec, par la présence du gouvernement fédéral avec ses fonctionnaires canadiens-français, par la disponibilité d'une abondante main-d'œuvre cléricale, Ottawa devint la capitale de l'Ontario français, le siège de presque toutes les associations franco-ontariennes d'envergure provinciale ${ }^{40}$. » L'historien souligne que « les deux comtés de Prescott et Russell sont la région française la plus homogène de 1'Ontario français ${ }^{41}$. »

\section{Les collections}

Le répertoire de l'Est nous provient de quatre sources principales. C'est ici que nous retrouvons les premières enquêtes folkloriques

39. Ibid, p. 49.

40. Gervais, « Les Paroisses de l'Ontario français 1767-2000», op. cit., p. 190.

41. Ibid. 
scientifiques en Ontario. Marius Barbeau, avec des collaborateurs comme É.-Z. Massicotte, Gustave Lanctot et Adélard Lambert, effectue des enquêtes des deux côtés de la rivière Outaouais à partir de 1917. Selon Jean-Pierre Pichette, ils vont recueillir 650 chansons auprès d'informateurs «d'origine franco-ontarienne ${ }^{42}$ »; je m'en suis tenu pour cette analyse aux 150 versions recueillies en Ontario et confirmées comme traditionnelles qui paraissent dans le Catalogue Laforte. Une deuxième source, la collection Cinésources 10, complètement inédite, est déposée au $\mathrm{CRCCF}^{43}$ et comprend une douzaine de rubans magnétiques contenant environ 300 chansons enregistrées dans divers centres culturels dans le comté de Prescott-Russell entre 1971 et 1977. L'écoute attentive en relève 154 qui sont de tradition orale. La troisième collection, la plus importante numériquement, est celle de Lucien Ouellet, ethnologue et ancien conservateur du programme de l'Amérique française au Musée canadien des civilisations (aujourd'hui Musée canadien de l'histoire). De 1986 à 1995, il recueillit, surtout à Ottawa et à Casselman, 565 chansons traditionnelles, qu'il identifia d'après la classification de Laforte, permettant ainsi une insertion facile dans ma banque de données provinciales. Chevauchant cette période, et presque aussi importante numériquement est la collection de 556 chansons recueillies sur ce même territoire par les étudiants du département de folklore de l'Université de Sudbury (Dfeus-eo) de 1981 à 2010. Enfin, le Catalogue Laforte rapporte pour cette région 203 chansons additionnelles de diverses collections, notamment celle de Paul Carpentier qui recueillit 177 chansons brèves dans les comtés unis de Prescott-Russell en 1970.

Le tableau 5 montre la distribution des chansons par catégorie dans chacune de ces collections ; le tableau 6 convertit ces chiffres en pourcentages.

42. Pichette, Répertoire ethnologique, op. cit., p. 25 et 28.

43. Centre de recherche en civilisation canadienne-française, fonds Cinésources (C7) M36.01, enregistrements $n^{\text {os }}$ S18/1/1 à S18/1/26. 


\section{Tableau 5}

Nombre total de types et de versions par catégorie dans les collections de l'Est ontarien

\begin{tabular}{|c|r|r|r|r|r|r|r|r|r|r|}
\hline $\begin{array}{c}\text { Catégorie } \\
\text { Laforte }\end{array}$ & \multicolumn{2}{|c|}{ Barb/Coll. } & \multicolumn{2}{|c|}{ Cinésources } & \multicolumn{2}{c|}{ Ouellet } & \multicolumn{2}{|c|}{ DFEus-Eo } & \multicolumn{2}{|c|}{ Autres } \\
\hline & $\mathrm{T}$ & $\mathrm{V}$ & $\mathrm{T}$ & $\mathrm{V}$ & $\mathrm{T}$ & $\mathrm{V}$ & $\mathrm{T}$ & $\mathrm{V}$ & $\mathrm{T}$ & $\mathrm{V}$ \\
\hline I. & 36 & 52 & 50 & 67 & 113 & 264 & 68 & 241 & 1 & 1 \\
\hline II. & 48 & 53 & 41 & 50 & 129 & 186 & 39 & 76 & 12 & 15 \\
\hline III. & 5 & 6 & 5 & 6 & 10 & 14 & 2 & 4 & 1 & 2 \\
\hline IV. & 11 & 17 & 14 & 17 & 45 & 63 & 38 & 109 & 7 & 7 \\
\hline V. & 11 & 16 & 1 & 1 & 5 & 7 & 43 & 79 & 64 & 177 \\
\hline VI. & 6 & 6 & 12 & 13 & 22 & 31 & 20 & 47 & 1 & 1 \\
\hline Total & $\mathbf{1 1 7}$ & $\mathbf{1 5 0}$ & $\mathbf{1 2 3}$ & $\mathbf{1 5 4}$ & $\mathbf{3 2 4}$ & $\mathbf{5 6 5}$ & $\mathbf{2 1 0}$ & $\mathbf{5 5 6}$ & $\mathbf{8 6}$ & $\mathbf{2 0 3}$ \\
\hline
\end{tabular}

\section{Tableau 6}

Pourcentage des catégories par collection dans l'Est ontarien

\begin{tabular}{|c|c|c|c|c|c|c|c|c|c|c|}
\hline $\begin{array}{c}\text { Catégorie } \\
\text { Laforte }\end{array}$ & \multicolumn{2}{|c|}{$\begin{array}{c}\text { Barb/ } \\
\text { Coll. }\end{array}$} & \multicolumn{2}{c|}{$\begin{array}{c}\text { Ciné- } \\
\text { sources }\end{array}$} & \multicolumn{2}{c|}{ Ouellet } & \multicolumn{2}{|c|}{ DFEus-EO } & \multicolumn{2}{|c|}{ Autres } \\
\hline & T & V & T & V & T & V & T & V & T & V \\
\hline I. & 30,8 & 34,7 & 40,7 & 43,5 & 34,9 & 46,7 & 32,4 & 43,4 & 1,2 & 0,5 \\
\hline II. & 41,0 & 35,3 & 33,3 & 32,5 & 39,8 & 32,9 & 18,6 & 13,7 & 14,0 & 7,4 \\
\hline III. & 4,3 & 4,0 & 4,1 & 3,9 & 3,1 & 2,5 & 0,9 & 0,7 & 1,2 & 1,0 \\
\hline IV. & 9,4 & 11,3 & 11,4 & 11,0 & 13,9 & 11,2 & 18,1 & 19,6 & 8,1 & 3,4 \\
\hline V. & 9,4 & 10,7 & 0,8 & 0,7 & 1,5 & 1,1 & 20,2 & 14,2 & 74,4 & 87,2 \\
\hline VI. & 5,1 & 4,0 & 9,8 & 8,4 & 6,8 & 5,5 & 9,5 & 8,5 & 1,2 & 0,5 \\
\hline
\end{tabular}

Plusieurs points à souligner ressortent de l'analyse des pourcentages. Je vais solliciter en particulier les chiffres représentant les pourcentages des versions (V), qui me paraissent les plus significatifs.

\section{Collection de Barbeau et de ses collaborateurs}

On peut considérer la collection de Barbeau et de ses collaborateurs, datant des premières décennies du siècle dernier, comme 
étalon pour évaluer les autres collections. On voit ici - comme dans les premières collections du Nord - un équilibre entre les deux formes majeures de chansons, les laisses et les strophiques, qui forment chacune un peu plus d'un tiers du répertoire $(34,7 \%$ et $35,3 \%$ respectivement). Ces chiffres s'accordent bien avec l'hypothèse que la chanson en laisse n'a pas toujours occupé la place primordiale dans le répertoire traditionnel qu'elle occupe aujourd'hui.

\section{Collections Cinésources et Ouellet}

Pour les collections qui se rapprochent de la fin du siècle - Cinésources et Ouellet - et surtout pour DFEus-Eo qui chevauche le début du $\mathrm{XXI}^{\mathrm{e}}$ siècle, l'écart est beaucoup plus grand, et on y retrouve effectivement les plus hautes proportions de laisses de toute la province. Dans la collection Ouellet, les versions de laisses constituent presque la moitié du répertoire $(46,7 \%)$. Ceci s'explique peut-être par le fait que l'informateur principal - l'étonnant Roger Lafrance, auprès de qui Lucien Ouellet a recueilli 244 chansons - avait été un homme de chantier, où ce genre était bien à l'honneur. La collection Cinésources, pour sa part, était aussi disposée à favoriser les chansons à répondre $(43,5 \%$ pour les laisses), puisqu'il ne s'agit pas dans ce dernier cas d'une enquête formelle, mais plutôt d'une série d'enregistrements effectués lors de rassemblements dans des centres communautaires ; la dynamique des groupes encourage évidemment la participation de l'assistance. Il y a quand même une portion significative de chansons strophiques exécutées par des participants exerçant un libre choix de pièces à chanter (32,5\%).

\section{Collection DFEUS-EO}

Comme dans le Nord, c'est la collection étudiante (DFEus-eO) qui apporte les perspectives les plus intéressantes. La proportion de chansons en laisse dans la collection $(43,4 \%)$ reflète celles des collections Cinésources et Ouellet, à partir d'une banque d'informateurs beaucoup plus étendue et variée. Les chansons 
strophiques, par contre, sont en chute libre dans ce répertoire, représentant seulement $13,7 \%$ des chansons chantées. Les chansons en forme de dialogue disparaissent complètement $(0,7 \%)$, c'est-à-dire 4 chansons sur $556^{44}$. Si on combine les chansons énumératives $(19,6 \%)$ avec les chansons en laisse, on constate que près des deux tiers $(63 \%)$ du répertoire sont composés de « chansons à répondre ». Comme leurs collègues du Nord, les étudiants de 1'Est accordent aussi une place significative aux chansons brèves dans leur répertoire (14,2\%). On pourrait supposer que les chansons brèves sont celles qui persistent le plus longtemps dans la mémoire - justement parce qu'elles sont brèves - mais aussi à cause que, dans bien des cas, ce sont les premières chansons que les informateurs ont apprises. Il est intéressant de noter que, au fur et à mesure que les chansons narratives disparaissent, la place relative des chansons brèves augmente.

Enfin la colonne «Autres » comprend surtout la collection de Paul Carpentier, soit 177 versions de 63 chansons brèves recueillies dans le cadre d'une recherche portant spécifiquement sur ce genre. La collection n'est donc pas représentative du répertoire général ; malgré la valeur de cette étude qui confirme la présence de nombreuses chansons brèves sur le territoire ontarien, il ne faudrait pas prendre ces données comme indication de la place réelle qu'elles occupent dans le répertoire. Cette étude est qualitativement différente des autres enquêtes de cette analyse et ses données viennent en quelque sorte fausser les résultats généraux ; elles seront donc retirées de l'analyse finale.

Il est évident que le répertoire de l'Est ontarien est celui qui est le plus proche de la province de Québec du point de vue géographique, démographique et culturel ; ceci soulève la possibilité que les changements dans le répertoire qui se produisent sur le plan diachronique ont peut-être aussi une composante géographique relative à leur proximité des sources québécoises.

44. Les quatre chansons incluent trois versions de «Frère Siméon », déjà mentionnée dans la note 33 . 


\section{Le sud de l'Ontario}

Cette région complexe se distingue des autres régions de la province à plusieurs égards, notamment en ce qui concerne la diversité de sa population et la position extrêmement minoritaire qu'occupe la plupart de ses membres. Dans le découpage provincial, elle renferme les plus anciens Franco-Ontariens et les plus récents. L'Office des affaires francophones sépare le Sud en deux parties : " le Centre » comme la région du grand Toronto et du Niagara et qui s'étend de Trenton jusqu'à Fort Érié, tout en englobant la Baie-Géorgienne ; « le Sud-Ouest », pour sa part, s'étend de London jusqu'à la frontière du Michigan ${ }^{45}$. Selon Yves Frenette, les régions partagent cependant suffisamment de caractéristiques communes pour permettre de discerner « l'éclosion d'une conscience régionale sur tout le territoire englobant les deux régions, en voie de devenir le Centre-Sud-Ouest ${ }^{46}$. " Mais du point de vue des traditions orales, il semble nécessaire de maintenir certaines distinctions entre le Sud-Ouest et le Centre.

Pour les besoins de cette étude, le Sud-Ouest se limite essentiellement à la ville de Windsor et aux comtés d'Essex et de Chatham-Kent, et correspond au territoire canadien de l'ancienne colonie du Détroit, fondée en 1701. Un bref survol historique confirmera le caractère distinct de cette région et son importance pour l'étude des traditions orales. La colonie originale, centrée autour du fort Pontchartrain de Détroit, incluait les terres des deux côtés de la rivière Détroit et attira jusqu'à la conquête britannique voyageurs, marchands et colons français et canadiens ; ce lieu fut le point stratégique et commercial le plus important entre Montréal et Québec et les possessions françaises dans la vallée du Mississippi. L'immigration de la vallée du Saint-Laurent continua

45. Office des affaires francophones, Profil statistique. Les francophones en Ontario, Toronto, Office des affaires francophones, s.d., p. 1.

46. Yves Frenette, «L'Ontario français du Centre et du Sud-Ouest, 19401970 », dans Cahiers Charlevoix. Études franco-ontariennes 7, Sudbury, Société Charlevoix et Prise de Parole, 2006, p. 147. Voir aussi Frenette, «Aspects de l'histoire des Franco-Ontariens du Centre et du Sud-Ouest, 1970-2000 », dans Cahiers Charlevoix. Études franco-ontariennes 10, Ottawa, Les Presses de l'Université d'Ottawa, 2014, p. 211-254 pour la suite de cette discussion. 
après l'arrivée des Anglais en 1760 et l'importance de la traite des fourrures diminua graduellement en faveur de l'agriculture. La rive nord passa sous juridiction étatsunienne en 1796. Longtemps avant la fin du XIX ${ }^{\mathrm{e}}$ siècle, les francophones de ce côté de la frontière devinrent minoritaires et les derniers vestiges de la présence francophone disparaissent au cours du $\mathrm{Xx}^{\mathrm{e}}$ siècle.

Du côté canadien, les francophones de la rivière Détroit sont bien établis depuis 1749 et en 1830 constituent encore la seule population de langue française permanente parmi les 100000 habitants anglophones du futur territoire ontarien. Vers 1840, une nouvelle vague d'immigration de la vallée laurentienne se dirige vers le Sud-Ouest à partir d'une crise économique au Bas-Canada ; ce mouvement, qui se prolonge jusqu'à la fin du XIX ${ }^{\mathrm{e}}$ siècle, voit la création de plusieurs paroisses francophones à l'est de Windsor, le long du lac Sainte-Claire. Ces nouveaux arrivants ont un engagement particulier envers l'agriculture et amènent avec eux une forte identité canadienne-française ${ }^{47}$. Les relations entre les établissements de la rivière Détroit et ceux du lac Sainte-Claire demeurent relativement restreintes et chaque population maintient des traits culturels particuliers ; les différences dans les traditions orales et le lexique employé par les deux groupes a fait l'objet de ma thèse de doctorat. Je ne reprendrai pas ces propos ici, mais la conclusion principale concernant les chansons montrait que le répertoire du premier groupe était très distinct de celui du deuxième groupe, qui avait plutôt tendance à se conformer au répertoire « laurentien » qui allait s'implanter ailleurs en province.

Le $\mathrm{xx}^{\mathrm{e}}$ siècle verra l'industrialisation des deux côtés de la rivière et une croissance économique et démographique remarquable. Les pressions d'assimilation - culturelle aussi bien que linguistique - sont très fortes pour la minorité francophone, ce qui est reflété par les taux d'assimilation les plus élevés de toute

47. Voir Jean Lapointe, «L'Historiographie et la construction de l'identité ontaroise », dans Simon Langlois, Identité et cultures nationales. L'Amérique française en mutation, Sainte-Foy, les Presses de l'Université Laval, 1995, p. 153-166 ; voir aussi Gaétan Gervais, «L'Ontario français (1821-1910) », op. cit., p 78-81, pour le contexte du peuplement dans le Sud-Ouest. 
la province. Le manque de médias francophones jusqu'en 1970 contribua sans doute à l'isolement de cette population des autres centres francophones de l'Ontario ; la faiblesse des liens encourage aussi une tendance conservatrice évidente dans le répertoire de chansons et le lexique, surtout parmi les francophones descendant du premier groupe colonisateur.

Malgré ce contexte sociohistorique unique, aucun folkloriste ne s'était penché sur cette région avant que je ne commence mes enquêtes en $1988^{48}$. Si le père Lemieux peut parler de Sudbury comme d'un "carrefour » où se rencontrent les folklores du Québec et de l'Ouest canadien ${ }^{49}$, on peut dire que le Détroit était aussi un carrefour où se côtoyaient Français, Canadiens, Anglais et Premières Nations, mais un carrefour qui devint effectivement un cul-de-sac à la fin du XVIII ${ }^{\mathrm{e}}$ siècle, en particulier pour les descendants du premier groupe. En conséquence, cette population évolua à l'abri des développements culturels ayant cours dans les autres régions francophones de l'Amérique. Les traditions orales préservées par les communautés de la rivière Détroit offrent donc des perspectives uniques sur le patrimoine oral de l'Amérique française.

La Huronie, du point de vue historique, a plus de points communs avec le Sud-Ouest qu'avec le Centre. Cette région au sud de la baie Géorgienne est en fait la deuxième région en Ontario colonisée par des francophones et les débuts de son peuplement précèdent eux aussi la grande diaspora québécoise. À partir de 1828, un groupe de voyageurs décident de suivre la garnison britannique lorsque celle-ci quitte l'île Drummond qui est cédée aux États-Unis. Ils s'établissent sur des terres octroyées à l'ouest de ce qui est aujourd'hui Pénétanguishene. Comme au Détroit, cette population initiale sera augmentée par plusieurs vagues d'immigration de la vallée du Saint-Laurent dans les décennies

48. Exception faite pour les études de Joseph-Médard Carrière qui recueillit vers 1938 vingt-six contes chez des informateurs de la région de Windsor et Tecumseh ; la collection fut publiée par Marcel Bénéteau et Donald Deschênes (dir.), Contes du Détroit, Sudbury, Prise de parole, 2005, 312 p.

49. Lemieux, Chanteurs franco-ontariens..., op. cit., p. 15. 
qui suivent; bon nombre de colons de la région de Batiscan et de Joliette s'établissent comme agriculteurs dans les cantons de Tiny et Tay pendant les années 1840 et 1850 . Un autre peuplement francophone du lac Huron, la région de Saint-Joseph dans le comté de Lambton, bien que relié à ce mouvement, est plus proche géographiquement de la région du Détroit et sera traité ainsi dans cette étude.

Le Centre, de son côté, abrite une population francophone beaucoup plus récente et hétérogène. Frenette souligne sa composition variée, contrairement à celle des communautés de l'Est et du Nord : « française, canadienne, métisse, québécoise, acadienne et, plus récemment, africaine et asiatique. Leur identité a donc été plus fragmentée qu'ailleurs... ${ }^{50}$ » Le territoire manque de cohérence interne et comprend la région du grand Toronto et de nombreuses autres localités qui abritent une minorité francophone importante, comme Welland, Hamilton, London et Sarnia (bien que cette dernière communauté soit plus proche du Sud-Ouest, sur le plan géographique). La région comprend aussi des francophones dispersés dans des centres n'ayant aucune collectivité francophone appréciable. Bien que les historiens, linguistes et sociologues se soient penchés plus récemment sur des questions identitaires concernant cette population nouvelle et diverse, du point de vue ethnologique elle n'a fait l'objet d'aucune étude systématique sur les traditions orales; les données rassemblées par les étudiants du DFeus seront donc nos seules références sur cette région.

\section{Les collections}

Comme je l'ai mentionné ci-haut, le Sud ontarien n'a pas attiré l'attention des folkloristes, qui se sont pour la plupart penchés sur les traditions de la diaspora canadienne-française du Québec. Quatre collections ont retenu mon attention pour la présente analyse. Ma propre enquête, initiée en 1988, se transforma en

50. Yves Frenette, «L'Ontario français du Centre et du Sud-Ouest, 1940-1970», op. cit., p. 147. 
recherche doctorale qui se prolongea jusqu'en 2001, année du tricentenaire de la présence française au Détroit du lac Érié. Le corpus fut rassemblé à partir de sources orales (65 informateurs natifs de la région), écrites (une vingtaine de chansonniers manuscrits) et imprimées (mémoires historiques, journaux et monographies paroissiales); bien que les deux groupes colonisateurs du Sud-Ouest y soient représentés, le répertoire du premier groupe compte pour environ deux tiers de la collection. C'est de loin la collection la plus importante du Sud-Ouest, avec 1705 versions de 635 chansons types ; elle exerce évidemment un poids considérable sur les données régionales.

Une seule collection nous parvient de la Huronie, une autre région négligée par les folkloristes. Louise Cadeau, Denise Jaïko et Daniel Marchildon ont interviewé une trentaine d'informateurs des cantons de Tiny et de Tay dans le cadre d'un projet d'été sur le folklore de la région, parrainé par le Centre d'activités françaises de Pénétanguishene en 1980. La collection, que j'ai pu consulter au $\mathrm{CRCCF}^{51}$, ajoute 68 versions de 64 chansons traditionnelles au répertoire franco-ontarien ; à celles-ci, j'ai ajouté 19 chansons de sources diverses.

Les étudiants du département de folklore de l'Université de Sudbury ont aussi fait des enquêtes dans le Sud ontarien. Pour les besoins de l'analyse, j'ai divisé ce corpus en deux unités : une pour le Sud-Ouest (Dfeus-so, 113 chansons) et l'autre pour le Centre (Dfeus-CE, 253 chansons). La collection DFEus-so présente des chansons recueillies sur le même terrain que celles de ma propre collection, mais auprès d'informateurs qui, pour la plupart, ne sont pas natifs de la région. L'enquête ne tient aucunement compte de la dimension historique et fournit donc des points de comparaison intéressants avec la mienne. La collection du Centre, pour sa part, nous donne un échantillon d'un corpus encore moins homogène et avec très peu d'associations régionales. Elle suscitera de nouvelles perspectives sur l'adaptation du répertoire dans un nouveau territoire.

51. CRCCF, collection C75. 
Les deux tableaux qui suivent présentent, respectivement, le nombre total de types et de versions, par collection, recueillis dans le sud de l'Ontario et les proportions des catégories Laforte dans le répertoire.

\section{Tableau 7}

Nombre total de types et de versions par catégorie dans les collections du Sud ontarien

\begin{tabular}{|c|c|c|c|c|c|c|c|c|}
\hline $\begin{array}{c}\text { Catégorie } \\
\text { Laforte }\end{array}$ & \multicolumn{2}{|c|}{ Bénéteau } & \multicolumn{2}{c|}{ Huronie } & \multicolumn{2}{c|}{ DFEus-so } & \multicolumn{2}{c|}{ DFEus-CE } \\
\hline & $\mathrm{T}$ & $\mathrm{V}$ & $\mathrm{T}$ & $\mathrm{V}$ & $\mathrm{T}$ & $\mathrm{V}$ & $\mathrm{T}$ & $\mathrm{V}$ \\
\hline I. & 88 & 278 & 18 & 23 & 25 & 41 & 36 & 66 \\
\hline II. & 348 & 999 & 35 & 38 & 10 & 11 & 14 & 16 \\
\hline III. & 47 & 111 & 7 & 7 & 2 & 2 & 7 & 9 \\
\hline IV. & 62 & 157 & 8 & 11 & 16 & 45 & 24 & 59 \\
\hline V. & 39 & 55 & 5 & 6 & 5 & 6 & 44 & 88 \\
\hline VI. & 55 & 105 & 10 & 12 & 6 & 8 & 10 & 15 \\
\hline Total & $\mathbf{6 3 9}$ & $\mathbf{1 7 0 5}$ & $\mathbf{8 3}$ & $\mathbf{9 7}$ & $\mathbf{6 4}$ & $\mathbf{1 1 3}$ & $\mathbf{1 3 5}$ & $\mathbf{2 5 3}$ \\
\hline
\end{tabular}

\section{Tableau 8}

Pourcentage des catégories par collection dans le Sud ontarien

\begin{tabular}{|c|c|c|c|c|c|c|c|c|}
\hline $\begin{array}{c}\text { Catégorie } \\
\text { Laforte }\end{array}$ & \multicolumn{2}{|c|}{ Bénéteau } & \multicolumn{2}{c|}{ Huronie } & \multicolumn{2}{c|}{ DFeus-so } & \multicolumn{2}{|c|}{ DFEus-CE } \\
\hline & $\mathrm{T}$ & $\mathrm{V}$ & $\mathrm{T}$ & $\mathrm{V}$ & $\mathrm{T}$ & $\mathrm{V}$ & $\mathrm{T}$ & $\mathrm{V}$ \\
\hline I. & 13,8 & 16,3 & 21,7 & 23,7 & 39,1 & 36,3 & 26,7 & 26,1 \\
\hline II. & 54,4 & 58,6 & 42,2 & 39,2 & 15,6 & 9,7 & 10,4 & 6,3 \\
\hline III. & 7,4 & 6,5 & 8,4 & 7,2 & 3,1 & 1,8 & 5,2 & 3,6 \\
\hline IV. & 9,7 & 9,2 & 9,6 & 11,3 & 25,0 & 39,8 & 17,8 & 23,3 \\
\hline V. & 6,1 & 3,2 & 6,0 & 6,2 & 7,8 & 5,3 & 32,6 & 34,8 \\
\hline VI. & 8,6 & 6,2 & 12,0 & 12,4 & 9,4 & 7,1 & 7,4 & 5,9 \\
\hline
\end{tabular}

\section{Collection Marcel-Bénéteau}

Mon intérêt principal pour dresser cette collection était d'abord celui d'un musicien à la recherche d'un répertoire local ; cependant, dès mes premières enquêtes, je vis le besoin de combler 
une lacune dans l'ensemble des grandes collectes folkloriques de l'Amérique du Nord. À mon avis, du point de vue historique et ethnologique, on avait tout simplement laissé de côté une des régions les plus significatives de l'Amérique française.

Quelques notes personnelles serviront de mise en contexte. Ayant moi-même grandi à Rivière-aux-Canards, dans une paroisse majoritairement francophone, mes connaissances sur la chanson traditionnelle étaient pourtant essentiellement limitées aux cahiers de La Bonne chanson $^{52}$; je n'ai jamais connu les veillées chantées qui étaient supposément caractéristiques de la communauté franco-ontarienne. Pour des raisons économiques, démographiques et culturelles, la pratique avait bel et bien disparu dans le Sud-Ouest à la fin de la Deuxième Guerre mondiale. En raison de l'industrialisation, le travail dans les usines avait généralement remplacé l'agriculture comme occupation principale et même ceux qui maintenaient la terre ancestrale accroissaient le revenu familial avec des quarts de travail dans les « facteries ». Les longues périodes hivernales d'activité réduite - la période des carnavals dont profitait la communauté agricole pour organiser veillées et soirées - ne trouvaient plus leur place dans le rythme accéléré de la vie moderne. La plupart des familles comptaient de plus en plus de membres non-francophones, facteur qui ne favorisait pas la transmission d'un répertoire français lors des rassemblements familiaux. La puissance médiatique de Détroit ${ }^{53}$ orienta le monde des loisirs et de la musique principalement autour du modèle étatsunien ; 1'obtention d'un poste de radio de RadioCanada en 1970 arrivait trop tard pour changer les habitudes d'écoute et de consommation de bien des gens.

52. Charles-Émile Gadbois, La Bonne Chanson, Saint-Hyacinthe, Éditions de la Bonne Chanson, 1938-1954, 10 vol. Cette série, diffusée à grande échelle dans les écoles et foyers francophones et considérée par plusieurs comme représentative du folklore canadien-français, comprend effectivement très peu de chansons de tradition orale ; en plus, elles sont généralement en versions standardisées et épurées.

53. Cette influence s'imposa très tôt : le poste radiophonique Wwo à Détroit commence à diffuser des émissions en 1920, un mois avant KDKA à Pittsburg; ce dernier est cependant le premier à obtenir un permis commercial. Les deux réclament aujourd'hui le titre de premier poste radiophonique au monde. 
On ne peut prétendre que ces facteurs sont exclusifs au SudOuest, mais, très tôt, ils ont eu un impact puissant sur cette région et expliquent en partie les faits observés : la transmission d'un répertoire traditionnel typique de la région s'était à toute fin pratique arrêtée avec la génération de mes parents, génération qui en avait quand même conservé le souvenir.

Il faut donc souligner que mes enquêtes n'étaient pas une recherche sur l'état actuel de la chanson traditionnelle dans le Sud-Ouest, mais plutôt un effort d'archéologie de la mémoire qui avait comme but de récupérer le répertoire traditionnel du Détroit que les Marius Barbeau et Germain Lemieux n'avaient pas documenté. À quelques exceptions près, mes informateurs étaient des personnes nées dans la région au cours des deux premières décennies du $\mathrm{xx}^{\mathrm{e}}$ siècle; dans le cas de mes informateurs de la rivière Détroit, il s'agissait de membres de familles établies dans la région depuis plus de deux siècles. Plusieurs d'entre eux avaient en leur possession des cahiers manuscrits où était inscrit leur répertoire familial. Cinq de ces cahiers de la rivière Détroit datent du tournant du $\mathrm{Xx}^{\mathrm{e}}$ siècle (1895-1910) et contiennent plus de 500 versions d'environ 350 chansons types attestées avant les premières enquêtes folkloriques de Marius Barbeau. Un bon nombre d'entre elles s'avèrent donc les plus anciennes attestations en Ontario, et même en Amérique du Nord. La collection complète compte 1705 versions de 639 chansons types, dont environ deux tiers proviennent de la rivière Détroit ; le reste a été collecté dans les communautés du lac Sainte-Claire (familles établies dans la deuxième moitié du XIX ${ }^{\mathrm{e}}$ siècle). Bien qu'il y ait des différences marquées entre les répertoires des deux groupes colonisateurs, dans un premier temps la collection sera traitée dans son ensemble ${ }^{54}$.

La typologie de cette collection se distingue immédiatement de toutes les autres collections que nous avons vues jusqu'ici. On

54. Voir Bénéteau, «Aspects de la tradition orale... », op. cit., pour une discussion approfondie des écarts. Voir aussi mon article « Chansons traditionnelles et identité culturelle chez les Francophones du Détroit » dans Ethnologies, vol. 26, n 2 , 2004, p. 201-220. 
note en premier lieu (tableaux 7 et 8 ), le nombre très faible de chansons en laisse : 88 chansons types sur un total de 639, soit $13,8 \%$ des chansons recueillies. Les versions totales ne comptent que pour $16,3 \%$ du répertoire - proportions étonnantes si on considère la place importante que cette catégorie de chansons occupe dans les répertoires du nord et de l'est de la province. Encore plus surprenante est la proportion des chansons strophiques : 348 chansons types sur un total de 639 (plus de la moitié) et 999 versions sur un total de 1705 , soit 58,6\% du répertoire. Les chansons en forme de dialogue, pour leur part, sont mieux représentées ici que dans toute autre collection ontarienne : $6,5 \%$ des versions recueillies appartiennent à cette catégorie. En d'autres mots, les deux tiers de la collection consistent en chansons narratives $(66 \%)$; les chansons « à répondre », combinant les laisses et les chansons énumératives, comptent seulement pour $25,5 \%$ du répertoire. Les chiffres indiquent une préférence certaine pour les chansons narratives sur le plus ancien territoire de l'Ontario français.

L'apport des cahiers manuscrits entre certainement en jeu dans cet inversement des données (bien que les proportions se maintiennent, de façon moins prononcée, même si on s'en tient uniquement aux versions chantées). Il faut toutefois noter que c'est la seule collection à reposer de façon aussi importante sur ce genre de source. Les cahiers manuscrits ont longtemps posé des problèmes aux ethnologues ${ }^{55}$. Ils représentent évidemment un choix de chansons sur lequel le chercheur n'a aucun contrôle et qui repose sur des critères différents de ceux employés par les ethnologues. Les cahiers contiennent toutes sortes de chansons et leurs compilateurs ne distinguent aucunement les pièces tradi-

55. Pour une discussion probante de cette question, voir Patrick Mazellier, « D'une collecte dans les Hautes Alpes aux cahiers de chansons correspondants : un bref éclairage sur l'importance des supports manuscrits et de leur utilisation dans les répertoires populaires chantés », et Claude Ribouilliault, "Les Cahiers de chansons manuscrits : aide-mémoire de l'oralité et contre-livres ? Sens et significations, essai de typologie », dans Jany Rouger (dir.), De l'écriture d'une tradition orale à la pratique d'une écriture. Actes du colloque de Clamecy (58) les 26 et 27 octobre 2000. Premières Rencontres autour de Achille Millien, Parthenay, Modal Éditions, 2001, p. 133-162 et 163-179. 
tionnelles des textes littéraires. Certains cahiers contiennent une proportion très élevée de chansons littéraires et laissent de côté les chansons bien connues du répertoire traditionnel : ce qu'on inscrit dans ces chansonniers sont les chansons rares, nouvelles ou obscures qu'on ne veut pas oublier. Selon le raisonnement de certains, les chansons «à répondre »- les plus populaires de toutes - n'ont donc pas besoin d'être transcrites. D'autres chercheurs ont avancé le point de vue que, avec son jeu complexe de refrains et de répétitions, la chanson en laisse est simplement trop difficile et lourde à transcrire pour des scripteurs semi-lettrés. Mais certains compilateurs - comme Félix Drouillard, fils d'agriculteur à Rivière-aux-Canards qui remplit son cahier avec 275 chansons entre 1897 et 1903 - semblent être de véritables chroniqueurs de ce qu'ils entendent dans leur milieu (Drouillard va jusqu'à noter le nom de ses « informateurs »). Son cahier contient 175 chansons de tradition orale française - parfois en versions différentes ainsi que 53 chansons littéraires et 47 chansons anglaises. La liste comprend toutes les chansons bien connues du répertoire régional, ainsi que des pièces rares ; comme je l'ai démontré ailleurs, il accomplit aussi très bien la transcription des chansons en laisse ${ }^{56}$. Il n'en demeure pas moins qu'il note seulement six laisses parmi les 175 chansons traditionnelles qu'il transcrit ! Il y a sans doute d'autres facteurs à considérer, mais le lecteur doit au moins admettre la possibilité que la chanson « à répondre » dans ce temps et lieu soit loin d'être la forme privilégiée qu'elle sera ailleurs en Ontario.

Je reviendrai dans mes considérations finales sur les raisons de cette divergence, mais je voudrais souligner que le terme même « chanson à répondre » était inconnu par la plupart de mes informateurs. Ils qualifiaient certaines chansons de « chansons à bras » (parce qu'on se les passait d'une personne à l'autre) ou simplement de « chansons qu'on faisait chorus », mais il pouvait aussi bien s'agir de chansons strophiques que de laisses.

56. Voir Marcel Bénéteau, « Le Chansonnier manuscrit comme document ethnologique. Considérations sur le cahier de Félix Drouillard (vers 1897-1903) », dans Rabaska, no 1, 2003, p. 61-78. 
À mon avis, les cahiers manuscrits - et surtout ceux de la période 1895-1910, à laquelle appartient le cahier de Félix Drouillard - fournissent un échantillon fiable du répertoire chanté dans la plus ancienne communauté francophone de l'Ontario, le seul échantillon qu'on puisse considérer bien implanté sur le territoire avant la fin du XIX $X^{e}$ siècle. Ce répertoire, transmis et maintenu en grande mesure à l'écart du répertoire « laurentien » qui s'imposa ailleurs en province, partage plusieurs des caractéristiques du répertoire des Acadiens, des Métis de l'Ouest et des anciens peuplements français du Centre-Ouest étatsunien - toutes des régions établies avant la diaspora québécoise du XIX $x^{e}$ siècle.

Le répertoire du Sud-Ouest est aussi très riche au niveau des chansons types individuelles. Non seulement les premières attestations ontariennes, mais les raretés y abondent : nous y retrouvons plus d'attestations uniques en Amérique du Nord et plus de nouvelles chansons que dans toute autre collection en Ontario : 90 chansons non attestées ailleurs dans la francophonie, 12 inventoriées par Coirault en France, mais absente du Catalogue Laforte ainsi que quatre que Laforte relève en France mais pas en Amérique française. Cette collection ajoute donc de façon considérable à la richesse du répertoire franco-ontarien.

\section{Collection Huronie}

Numériquement, cette collection n'apporte pas beaucoup d'information ; en plus des chansons recueillies au Centre d'activités françaises en 1980, j'y ai ajouté les quelques versions de la collection Hélène Desroches qui figurent dans le Catalogue Laforte et d'autres recueillies par les étudiants de l'Université de Sudbury pour en arriver à 97 versions de 83 chansons types. Mais comme seul échantillon de cette région historique, il est intéressant de noter que la typologie reflète d'assez près les proportions du corpus du Sud-Ouest : un taux relativement faible de chansons en laisse ( $23,7 \%$ des versions totales) et un taux élevé de chansons strophiques (39,2\%: seulement Lemieux et Bénéteau rapportent plus de chansons strophiques). Comme dans le réper- 
toire du Sud-Ouest, les versions de chansons narratives $(46,4 \%)$ occupent une place plus importante que celle des chansons «à répondre » $(35 \%)$.

\section{Collections DFEUS}

Les deux colonnes portant sur les collections étudiantes dans le Sud-Ouest et le Centre présentent un contraste assez étonnant avec les autres collections issues de cette région. Commençons par les chansons recueillies dans le Sud-Ouest (DFEus-So), exactement sur le même terrain où j'ai puisé ma collection. Quoique la quantité de chansons recueillies ne soit pas significative (113 versions de 64 chansons types), l'écart dans les proportions ne pourrait pas être plus marqué : les chansons en laisse $(36,3 \%)$ et les chansons énumératives $(39,8 \%)$ représentent $76,1 \% \mathrm{du}$ total pour les « chansons à répondre », soit plus des trois quarts du répertoire, le taux le plus élevé dans toute la province. Les chansons narratives (strophiques 9,7\% et dialoguées 1,8\%) donnent $11,5 \%$ du répertoire, soit le taux le plus faible de toute la province. Les chansons recueillies dans le Centre (DFEus-CE), un peu plus nombreuses (253 versions de 135 chansons types) se répartissent selon des pourcentages similaires avec une exception notable : ici, la proportion occupée par les chansons brèves $(34,8 \%)$ est assez élevée pour faire baisser la part des chansons en laisse (26,1\%). Plusieurs des chansons brèves recueillies ici semblent avoir été apprises à l'école, ce qui indiquerait davantage une transmission institutionnelle que communautaire.

Ces deux petites collections suggèrent des tendances intrigantes. Pour le Sud-Ouest, la plupart des informateurs ne semblent pas être natifs de la région (on y retrouve très peu des patronymes typiques du Sud-Ouest) ; pour le Centre, nous savons que la population francophone est plutôt récente. On peut donc supposer que ces informateurs n'ont pas de racines profondes dans leurs communautés et qu'un certain contexte nécessaire à la transmission du répertoire traditionnel est absent : les occurrences de réunions de familles multigénérationnelles, de mariages et noces endogames, 
et d'autres rassemblements de francophones partageant une culture commune sont sans doute plus rares pour des gens arrivant dans une communauté, ce qui suggère que la transmission orale n'est pas la source principale du répertoire.

Deux projets de recherche ont tenté d'expliquer l'évolution des pratiques culturelles traditionnelles des populations dans des régions éloignées des grands centres culturels. Jean-Pierre Pichette, avec la théorie du limaçon et de la « résistance des marges » souligne le fait que les traditions se maintiennent souvent mieux dans la périphérie ${ }^{57}$ que dans les grands centres ; son étude sur la « danse sur les bas » en est un exemple frappant. Gérard Bouchard et son équipe de l'IREP (Institut interuniversitaire de recherche sur les populations) postulent le contraire : qu'il se produit une « érosion » de la culture avec perte des pratiques traditionnelles lors des déplacements de population ${ }^{58}$. Je dirais que la première hypothèse s'applique très bien dans une communauté plus ou moins stable, homogène et refermée sur elle-même, comme le Nord ontarien de Germain Lemieux ; n'oublions pas qu'à l'époque de ses enquêtes, la paroisse demeurait l'institution principale des francophones et que les contacts avec l'extérieur étaient assez rares. La situation était semblable dans le SudOuest où, malgré la proximité des centres urbains, la plupart de mes informateurs avaient grandi dans des communautés rurales francophones, catholiques et endogames. Mais là où le support institutionnel et la masse critique n'existent pas pour la transmission et le maintien des anciennes traditions, on peut certainement envisager une certaine érosion de celles-ci.

Un patrimoine oral évolue dans un certain contexte, dans un environnement particulier. Ce n'est pas tout le bagage culturel qu'un immigrant amène qui s'avère utile dans un nouvel envi-

57. Jean-Pierre Pichette, «Le Principe du limaçon ou la résistance des marges. Essai d'interprétation de la dynamique des traditions ", dans les Cahiers Charlevoix. Études franco-ontariennes 8, Ottawa, Société Charlevoix et Presses de l'Université d'Ottawa, 2010, p. 11-75.

58. Voir surtout Gérard Bouchard et Martine Segalen (dir.), Dynamiques culturelles interrégionales au Québec et en France. Construction d'une enquête, Chicoutimi, Institut interuniversitaire de recherches sur les populations 1995, $260 \mathrm{p}$. 
ronnement, ce ne sont pas toutes les composantes qui peuvent être adaptées à un nouveau contexte. Les chansons narratives, plus longues et plus complexes, véhiculant les histoires et les valeurs du groupe, ont besoin d'un certain contexte social, d'un rythme de vie convenable, de l'appui générationnel de la famille étendue pour se maintenir dans un nouveau foyer. Les chansons qui voyagent le mieux sont les chansons comiques et entrainantes qui facilitent davantage la participation communautaire et la cohésion sociale. Le rôle des médias et du système scolaire n'est pas négligeable dans tout cela : les premiers, tout au cours du $\mathrm{Xx}^{\mathrm{e}}$ siècle, ont eu tendance à favoriser les chansons à répondre tandis que les cadres institutionnels ont été portés à valoriser une culture commune aux dépens du maintien de particularités régionales. La créativité et l'expression identitaire se manifestent aujourd'hui à travers d'innombrables autres médias, et le maintien et la manipulation d'un vaste répertoire de pièces remontant à l'ancienne France n'est plus le véhicule de choix. On garde certes quelques chansons faciles et immédiatement reconnaissables puisqu'elles font partie d'un patrimoine qu'on nous présente comme un autre produit à consommer. Il y a sans aucun doute un rétrécissement du répertoire sous ces conditions et l'effet est clairement visible dans les collections étudiantes du sud de l'Ontario.

\section{Considérations finales}

Pour la première fois, nous pouvons décrire avec précision l'envergure et la composition du répertoire de chansons traditionnelles française transmis et maintenu par la deuxième plus grande population francophone en Amérique du Nord. Ces descriptions ne s'appuient pas sur des extrapolations à partir d'autres répertoires, mais sur des données recueillies depuis la fin du XIX ${ }^{\mathrm{e}}$ siècle jusqu'au début du XXI ${ }^{\mathrm{e}}$ siècle sur un terrain qui comprend toutes les régions francophones de l'Ontario. Les chiffres bruts eux-mêmes étonnent : 12000 versions de presque 1500 chansons types, un corpus dont l'ampleur et la diversité ne permettront plus jamais 
à quiconque s'intéressant au patrimoine de l'Amérique française d'y passer outre.

Le répertoire multiplie les versions de chansons déjà connues, et en même temps ajoute des centaines de nouvelles chansons types aux répertoires ontarien, nord-américain et même d'outremer. Les données recueillies confirment la présence en Ontario de 690 chansons types répertoriées ailleurs par Laforte, mais pour lesquelles il n'a noté aucune attestation ontarienne. Ces données font augmenter de plus du double les 557 chansons types déjà identifiées par lui en Ontario. Mes recherches confirment d'ailleurs la présence ontarienne de 100 autres chansons types, clairement d'origine traditionnelle, déposées dans divers fonds d'archives mais absentes du Catalogue Laforte et du Répertoire Coirault. J'ai aussi identifié dans le répertoire ontarien 31 chansons répertoriées en France par Coirault qui sont complètement absentes du Catalogue Laforte ou relevées là uniquement dans des versions européennes. Enfin, les collections rapportent un total de 170 nouvelles chansons types jamais répertoriées ailleurs ${ }^{59}$.

Pour les chansons types déjà inscrites dans Laforte, les données multiplient les versions connues. L'importance de cet apport n'est pas simplement numérique : chaque version d'une chanson type apporte de nouvelles dimensions et contribue à notre appréciation de toutes les possibilités inhérentes à son scénario global. En voici trois exemples, choisis au hasard : aux 66 versions de la chanson en laisse bien connue Le Bateau chargé de blé (I. F-21) répertoriées par Laforte, il faut maintenant ajouter 123 nouvelles versions. Évidemment, il y a une certaine redondance dans le cas de cette chanson largement diffusée par l'entremise des cahiers de la Bonne Chanson (sous le titre « À Saint-Malo, beau port de mer »). Mais plusieurs des versions provenant des trois régions ontariennes mettent en valeur une forme alternative de la chanson, relativement rare au Québec, qui se chante sur un air et avec un

59. Je n'inclus pas dans ces chiffres les 123 chansons brèves non identifiées pour lesquelles je n'ai pu vérifier la répartition, pour les raisons déjà citées, ni les 78 chansons de composition locale pour lesquelles on ne pourrait s'attendre à une distribution qui s'étend au-delà du terrain de collecte immédiat. 
refrain qui sont très différents de ceux de la forme bien connue et qui offrent un scénario beaucoup plus développé. Ou prenons l'exemple d'une chanson strophique que Laforte classe sous le titre La Bouteille (II. D-4) : le Catalogue compte six versions québécoises seulement. L'examen de celles-ci révèle une structure qui semble chevaucher les formes strophiques et la laisse ; l'étude des cinq nouvelles versions ontariennes, plus complètes, montrent sans aucun doute qu'il s'agit effectivement d'une ancienne laisse répertoriée en France par Coirault sous le titre 4802 La belle courtisée par trois gentilshommes. Et comme troisième exemple, on retrouve dans Laforte cinq versions québécoises de la chanson Mes amis de plaisir (II. R-13), que l'ethnomusicologue Marc Gagné décrit ainsi : «Les strophes, souvent incohérentes, pourraient être une suite de bribes de chansons différentes et difficilement identifiables ${ }^{60} »$. Les huit nouvelles versions ontariennes présentent pourtant dans l'ensemble un scénario très bien dessiné d'une jeune fille déçue par son amant et qui s'adonne à de grandes beuveries pour soulager ses peines.

Je pourrais multiplier de tels exemples et, dans un article à suivre, je décrirai en plus grand détail les contributions majeures du répertoire franco-ontarien au patrimoine chansonnier. Chaque région met en vedette certaines chansons qui sont chantées exclusivement sur son propre territoire. Mais au-delà des chansons individuelles, l'analyse interne des répertoires a déjà relevé des divergences typologiques importantes sur les plans géographique et diachronique. Essentiellement, on peut dire que des chansons de catégories différentes prédominent dans certaines régions plus que d'autres. En s'appuyant sur la répartition des six catégories traditionnelles de Conrad Laforte - catégories qui se différencient non seulement par leur forme poétique mais aussi du point de vue de leurs thématiques générales et de leurs fonctions sociales -, des divergences très nettes se dégagent. Les graphiques de la figure 1 illustrent les proportions dans lesquelles les six catégories du

60. Marc Gagné et Monique Poulin, Chantons la chanson. Enregistrements, transcriptions et commentaires de chansons et de pièces instrumentales, Québec, Les Presses de l'Université Laval, 1990, p. 141. 
Catalogue Laforte sont présentes dans chaque répertoire; pour chaque région, les données de toutes les collections sont incluses sans tenir compte de l'aspect diachronique ou du poids relatif de chaque collection. Les données de Paul Carpentier portant exclusivement sur la chanson brève dans la région d'Ottawa ont néanmoins été omises afin de donner un portrait plus précis du répertoire de l'Est ontarien; notons que la part disproportionnée des chansons strophiques dans le tableau du Sud ontarien apparaît malgré les taux très faibles de cette catégorie dans les collections étudiantes.

\section{Figure 1}

Pourcentages des catégories Laforte dans le répertoire de chaque région

I. Chansons en laisse - II. Chansons strophiques

III. Chansons en forme de dialogue - IV. Chansons énumératives

V. Chansons brèves - VI. Chansons sur les timbres
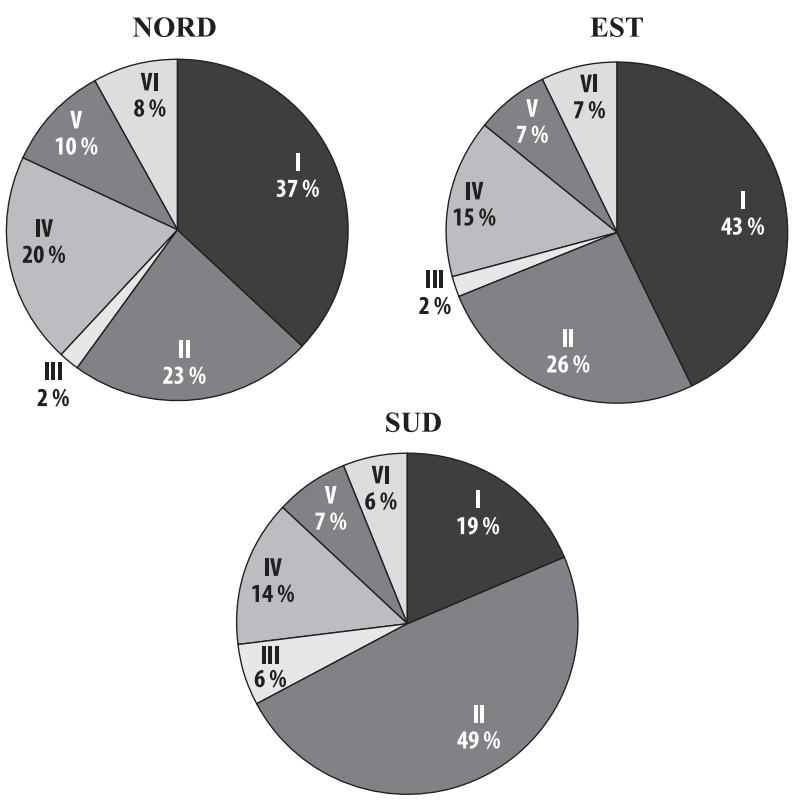

Au cours de mon analyse, j'ai souligné avant tout l'opposition de ce qu'on appelle communément les « chansons à répondre » (catégories I et IV) et les « chansons narratives » (catégories II 
et III). Ces désignations ne sont pas des termes scientifiques et ne relèvent pas de critères fixes : certaines chansons strophiques sont chantées avec la participation de "répondeurs » et même une chanson énumérative peut parfois raconter une histoire rudimentaire. Mais les deux genres correspondent cependant à deux fonctions complémentaires du répertoire traditionnel. À cet égard, on voit des ressemblances marquées dans la composition des répertoires du Nord et de l'Est : les deux affichent, à presque $60 \%$, leur préférence pour les chansons à répondre $(57 \%$ et $58 \%$ respectivement) et seulement un quart pour les chansons narratives $(25 \%$ et $28 \%)$. Le portrait pour le Sud, cependant, est complètement différent, affichant $55 \%$ de chansons narratives et $33 \%$ de chansons à répondre (et ceci même avec les données confondues du Sud-Ouest et du Centre).

Le Sud comme terrain se distingue des autres régions en raison de sa diversité démographique et un certain isolement géographique et culturel. Il comprend aussi le plus ancien peuplement francophone en Ontario. Ces facteurs sont-ils responsables les écarts observés ? Revenons aux trois régions : du point de vue géographique, l'est de l'Ontario est contigu à la province du Québec, formant une quasi-unité, non seulement dans la région de la capitale nationale, mais aussi en des points de contact comme la ville de Hawkesbury. Étant donné cette proximité et les liens évidents entre les deux rives et leurs fonds démographiques, il serait difficile de distinguer les répertoires d'une rive à l'autre. Des informateurs importants de l'Est ontarien, comme Roger Lafrance, semblent avoir passé autant de temps dans une province que dans l'autre. Une étude approfondie relèverait sans aucun doute des différences régionales au Québec comme en Ontario, mais on peut avancer que les proportions présentes dans les données que j'ai recueillies pour l'Est sont tout à fait conformes avec celles du répertoire « laurentien » défini par Jean-Pierre Pichette.

La population du Nord est aussi à plusieurs égards une continuation du foyer laurentien; bien que plus distante de sa terre d'origine (sauf dans la région du Témiscamingue), l'arrivée rela- 
tivement récente de ses habitants a longtemps favorisé le maintien des liens familiaux et culturels avec le Québec et l'Est ontarien. Ces liens sont beaucoup moins forts dans le Sud-Ouest, où les influences culturelles ont plutôt tendance à venir de l'Ontario anglais et des États-Unis.

Une première conclusion s'impose donc : plus on s'éloigne physiquement et culturellement du foyer laurentien, plus le répertoire a tendance à diverger de ce modèle. Cette observation semble aussi valable à mesure que l'on s'éloigne sur le plan diachronique. Les répertoires des trois régions ont été implantés sur leurs territoires à différentes époques et dans des contextes socioéconomiques différents. La chanson à répondre dans son ensemble est déjà plus populaire à l'époque du peuplement de l'Est et du Nord, mais on note que les collections les plus anciennes - celles de Barbeau et Lemieux, par exemple - témoignent d'une distribution assez équilibrée de laisses et de chansons strophiques, équilibre qui bascule complètement dans les collections plus récentes. À la fin $\mathrm{du} \mathrm{xx}^{\mathrm{e}}$ siècle, les « chansons à répondre » en viennent à prédominer dans les trois grandes régions de l'Ontario français.

Les éléments de preuve pour l'ascendance plutôt récente des chansons en laisse en particulier sont assez convaincants. Malgré leur grande popularité dans le répertoire du Québec, de l'Ontario et de la Nouvelle-Angleterre, on retrouve beaucoup moins de chansons en laisse dans les traditions de populations établies antérieurement à la diaspora québécoise du XIX ${ }^{\mathrm{e}}$ siècle, comme chez les Métis de l'Ouest canadien, les Acadiens et les Cadiens de la Louisiane ; il en est de même pour les quelques collections provenant d'anciens peuplements français du CentreOuest étatsunien. Les élites canadiennes-françaises ont tenté dès les premières publications de chansonniers de relier ce genre de chanson - longtemps associé aux chants de voyageurs et aux camps de bûcherons - au caractère « gai et joyeux » des Canadiens-français ; ce processus était déjà entamé à l'époque des migrations québécoises ${ }^{61}$. Les médias du $\mathrm{XX}^{\mathrm{e}}$ siècle n'ont fait

61. Pour l'influence du clergé sur le répertoire traditionnel, voir Marcel Bénéteau, 
qu'intensifier cette tendance. Le musicien et folkloriste québécois Michel Faubert, lors d'une émission de Radio-Canada et de la Radio suisse romande, discute de la médiatisation de la chanson folklorique à partir de Montréal durant les années 1920 ; selon lui, ce processus aurait « provoqué la perte de certains répertoires [...]. On mettait l'accent sur les chansons plus joyeuses, courtes, qui permettaient de faire la fête ${ }^{62} »$. Depuis cette époque, tout un volet de l'industrie du disque a continué à faire la promotion de ce genre de musique. Les répertoires des régions qui ont maintenu des liens avec la culture québécoise reflètent naturellement ce biais.

Un retour sur les trois collections principales - celles de Germain Lemieux, du Dfeus-No et ma propre collection du SudOuest - illustre très bien les changements sur le plan diachronique. Comparons d'abord les deux collections du Nord, recueillies, je le rappelle, de façon consécutive sur le même terrain.

\section{Figure 2}

Pourcentages des catégories Laforte dans les collections Lemieux et DFEUS-NO

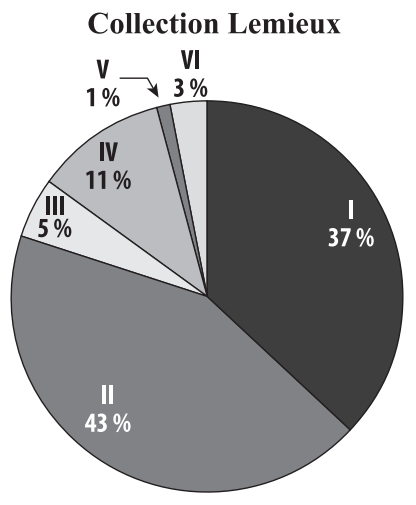

\section{Collection DFEus-No}

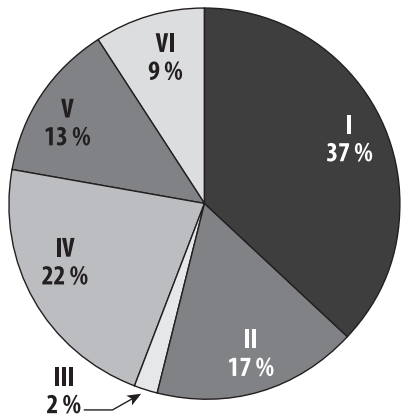

"Les Prêtres et religieux auteurs de chansonniers », Port Acadie, "L'Apport des prêtres et des religieux au patrimoine des minorités. Parcours comparés Bretagne/ Canada français ", $n^{\text {os }}$ 24-25-26, automne 2013, printemps 2014, été 2014, p. 259-272.

62. Société Radio-Canada, «La Suisse rencontre le Canada », émission spéciale de Des musiques en mémoire, diffusée par Radio-Canada et la Radio suisse romane, le 24 octobre 1994 ; discussion en table ronde avec Michel Faubert, Charlotte Cormier, Marcel Bénéteau et Jean Nicol. 
Dans la première collection, celle de Lemieux, on peut constater un équilibre parfait entre les chansons à répondre (I et IV) et les chansons narratives (II et III), c'est-à-dire $48 \%$ pour les deux genres de chansons. Dans la collection plus récente, l'équilibre n'est plus en évidence : on compte $59 \%$ pour les chansons à répondre et seulement $19 \%$ pour les chansons narratives. On observe donc, dans la période 1948 à 2010, une diminution nette des chansons narratives avec une augmentation correspondante des chansons à répondre. La collection Bénéteau, comprenant les plus anciennes données dans toute la province, vient élargir cet écart en accordant les deux tiers du répertoire aux chansons narratives et seulement un quart aux chansons à répondre.

\section{Figure 3}

Pourcentages des catégories Laforte dans la collection Bénéteau

Collection Bénéteau

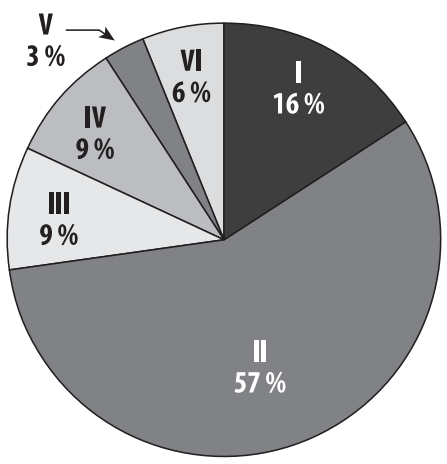

\section{Conclusion}

La définition de « chanson de tradition orale » n'inclut pas toute chanson populaire en voie de folklorisation, mais un ensemble bien précis - sinon canonique - de poésies chantées avec ses racines dans la France du XV ${ }^{\mathrm{e}}$ au XVIII ${ }^{\mathrm{e}}$ siècle (approximativement) et amené en Amérique du Nord lors de la colonisation de la Nouvelle-France où ce répertoire fut maintenu, adapté et élaboré 
par les descendants des colonisateurs des diverses régions. Que les gens continuent à chanter n'est pas remis en question - les 8000 versions des archives du département de folklore de l'Université de Sudbury en attestent résolument - et la chanson traditionnelle fait toujours partie de l'expression d'identité et d'appartenance des francophones de 1'Ontario. Mais à l'intérieur de ce fonds amené auX XVII ${ }^{\mathrm{e}}$ et $\mathrm{XVIII}{ }^{\mathrm{e}}$ siècles, il y a eu sans aucun doute une diminution dans le nombre et la diversité de chansons chantées. Les Franco-Ontariens chantent moins de chansons et moins de sortes de chansons qu'auparavant. Avec cette évolution disparaît tout un volet de thèmes et de scénarios traditionnels : amourettes de bergers et de bergères, aventures de soldats et de conscrits, thèmes religieux, épiques et tragiques.

Ce processus est sans doute le résultat du passage d'une culture orale à une culture lettrée devenue aujourd'hui numérique et ce n'est pas mon intention de faire le deuil de formes d'expressions qui n'ont plus cours dans la société contemporaine. Mais, tout comme la littérature écrite des siècles révolus maintient sa valeur dans la vie d'aujourd'hui, ce vaste corpus oral apporte aussi des éclairages sur notre histoire, notre culture, sur le comportement et la mentalité de nos ancêtres et sur leurs retombées dans les enjeux et les attitudes du monde contemporain. Il mérite d'être étudié en soi et valorisé aujourd'hui et à l'avenir. Il est à souhaiter que le dépistage et le classement des riches fonds de l'Ontario français facilite cette tâche. Nous devons notre gratitude à des générations de folkloristes et ethnologues - depuis les grands maîtres comme Barbeau et Lemieux, jusqu'au plus humble étudiant s'efforçant de remplir les exigences de ses professeurs - d'avoir sauvé de l'oubli ce précieux patrimoine. 